\title{
6d superconformal Cardy formulas
}

\author{
June Nahmgoong \\ Department of Physics and Astronomy \& Center for Theoretical Physics, \\ Seoul National University, \\ Gwanak-ro, Seoul 08826, Republic of Korea \\ E-mail: junenahmgoong@gmail.com
}

ABSTRACT: We study the superconformal index of $6 \mathrm{~d}$ SCFTs from their 't Hooft anomalies. In the Cardy limit where the angular momenta on $S^{5}$ are large, we show that the leading free energy, as well as a few subleading corrections, can be computed from the $6 \mathrm{~d}$ anomaly polynomials. Our large $N$ free energy accounts for the entropy of supersymmetric black holes in dual $A d S_{7}$.

Keywords: AdS-CFT Correspondence, Anomalies in Field and String Theories, Field Theories in Higher Dimensions

ArXiv EPrint: 1907.12582 


\section{Contents}

1 Introduction and summary 1

2 Thermal derivative expansion 4

2.1 Chern-Simons action and 't Hooft anomaly 5

2.2 6d index from Chern-Simons action 9

3 Cardy formulas of 6d SCFTs $\quad \mathbf{1 6}$

$3.1(1,0)$ SCFT without flavor chemical potentials 17

$\begin{array}{lll}3.2 & (2,0) \text { SCFT of ADE type } & 19\end{array}$

3.3 E-string theory of arbitrary rank 22

3.4 M5 branes on ALE singularities 24

4 Equivariant integral of the thermal anomaly polynomial 28

5 Asymptotic entropy $\quad 31$

5.1 Holographic SCFTs and $A d S_{7}$ black holes 31

5.2 Bound on the anomaly coefficients 34

6 Concluding remarks $\quad 35$

A $\quad$ A $4 d \mathcal{N}=1$ Cardy formula $\quad 36$

B Modified index of $6 \mathrm{~d}$ supermultiplets $\quad 38$

\section{Introduction and summary}

The superconformal index of a $d$-dimensional SCFT counts BPS states on $S^{d-1}$ [1-3]. For a 6d SCFT, its superconformal index is defined as

$$
\mathcal{I}=\operatorname{Tr}\left[(-1)^{F} \cdot e^{-\Delta_{R} Q_{R}} \cdot e^{-\omega_{1} J_{1}-\omega_{2} J_{2}-\omega_{3} J_{3}} \cdot \prod_{i} e^{-m_{i} F_{i}}\right]
$$

where $J_{1,2,3}$ are the angular momenta on $S^{5}, Q_{R}$ is the Cartan charge of SU(2) R-symmetry of $(1,0)$ superconformal algebra, and $F_{i}$ 's are the charges of the other global symmetries. Chemical potentials $\Delta_{R}$ and $\omega_{1,2,3}$ are constrained by $\Delta_{R}-\omega_{1}-\omega_{2}-\omega_{3}=0$, and only BPS states which saturate the BPS bound $E \geq 4 Q_{R}+J_{1}+J_{2}+J_{3}$ contribute to the index. The $6 \mathrm{~d}$ superconformal index was studied in [4-10] and see [11] for a detailed review.

6d SCFTs on $N$ M5-branes are expected to have $N^{3}$ degrees of freedom [12]. $N^{3}$ dependence of $6 \mathrm{~d}$ SCFTs are observed in the anomaly polynomials [13-15], the Casimir 
energy $[4,8-10,16]$, and $\mathbb{R}^{4} \times T^{2}$ partition function $[17,18]$. However, there has been no direct evidence for $N^{3}$ growth of states from the superconformal index.

As a related matter, it had been believed that the superconformal index could not capture the entropy of BPS black holes in the dual AdS space due to the severe boson/fermion cancellation. However, inspired by the extremization principle found in [19-21], it has been recently observed that the superconformal index can actually reproduce the entropy of supersymmetric black holes in $A d S_{4}$ [22-24], $A d S_{5}$ [25-27], $A d S_{6}$ [28], and $A d S_{7}$ [26]. The primary breakthrough was allowing the chemical potentials to be complex to obstruct $(-1)^{F}$ cancellation [29]. The complexified solution of the chemical potentials was recently obtained also in the gravity dual [30,31].

The Cardy formula of 2d CFT [32] plays a crucial role in accounting the entropy of black holes from field theories [33]. In $d>2$, the Cardy formula refers to the behavior of the superconformal index in the 'generalized Cardy limit' where $\left|\omega_{i}\right| \ll 1$. It corresponds to the limit where the angular momenta are large, and therefore it is in analogy with the limit considered in the original Cardy formula of 2d CFT. In [34], the Cardy formula of 4d SCFTs was studied with the strictly real chemical potentials. It was further studied in $[26,35-38]$ at the complex chemical potentials. It turns out that the free energy of the index captures $N^{2}$ growth, which accounts for the entropy of $A d S_{5}$ black holes.

The 6d Cardy formula was obtained in $[34,39]$ in the setting where $N^{3}$ growth is invisible. With the complex chemical potentials, a similar study of $6 \mathrm{~d}$ SCFTs was initiated by [26] for $(2,0) A_{N}$ theories in the large $N$ limit to reproduce the entropy of $A d S_{7}$ black holes. In this paper, we make comprehensive studies of various 6d SCFTs. In particular, we will complete the argument given in [26], and obtain Cardy formulas of various 6d SCFTs.

In this paper, we study the modified version of the superconformal index which is defined as follows,

$$
I=\operatorname{Tr}\left[e^{-\Delta_{R} Q_{R}} \cdot e^{-\omega_{1} J_{1}-\omega_{2} J_{2}-\omega_{3} J_{3}} \cdot \prod_{i} e^{-m_{i} F_{i}}\right]
$$

where chemical potentials are constrained by $\Delta_{R}-\omega_{1}-\omega_{2}-\omega_{3}=2 \pi i$. The modified index counts the same BPS states with the original index but on the different chemical potential basis. Note that $(-1)^{F}=e^{2 \pi i J_{3}}$ since $J_{I}$ 's are normalized to be integers for bosonic states and half-integers for fermionic states. Then, we can replace $(-1)^{F}$ in $(1.1)$ by $e^{2 \pi i J_{3}}$ and then absorb it into the redefinition of $\omega_{3}$ which shifts the chemical potential constraint. The modified index turns out to be more 'refined' in the sense that it can capture more entropy than the original index in the Cardy limit. Similar modification of the index in $4 \mathrm{~d}$ was studied in [37].

In order to compute the index (1.2), we consider the following thermal partition function on $S^{5} \times S^{1}$,

$$
Z=\operatorname{Tr}\left[e^{-\beta E} \cdot e^{-\Delta_{R} Q_{R}} \cdot e^{-\omega_{1} J_{1}-\omega_{2} J_{2}-\omega_{3} J_{3}} \cdot \prod_{i} e^{-m_{i} F_{i}}\right]
$$

where the periodicity of the temporal $S^{1}$ is given by $\beta$. The partition function (1.3) becomes the index (1.2) if one takes $\beta \rightarrow 0$ limit and imposes $\Delta_{R}-\omega_{1}-\omega_{2}-\omega_{3}=2 \pi i$ constraint [26]. 
The partition function can be computed from the effective action of background fields, which depend on the chemical potentials. In $\beta \rightarrow 0$ limit, the infinitely many terms in the effective action are arranged into a derivative expansion on $S^{5}$. Among them, ChernSimons terms can be entirely determined by the anomaly polynomials [40-42]. We will show that in the Cardy limit $\left|\omega_{i}\right| \ll 1$, the leading free energy of the index, as well as a few subleading corrections, are determined from the Chern-Simons action only.

For a general 6d SCFT, we find that the free energy $(\log I)$ of the modified index (1.2) with $m_{i}=0$ is given as follows,

$$
\begin{aligned}
\log I= & -\left(\frac{\mathfrak{a}}{384} \Delta_{R}^{4}+\frac{\mathfrak{b} \pi^{2}}{24} \Delta_{R}^{2}+\frac{2 \mathfrak{c} \pi^{4}}{3}\right) \frac{1}{\omega_{1} \omega_{2} \omega_{3}}+\left(\frac{\mathfrak{b}}{96} \Delta_{R}^{2}+\frac{(2 \mathfrak{c}+\mathfrak{d}) \pi^{2}}{6}\right) \frac{\omega_{1}^{2}+\omega_{2}^{2}+\omega_{3}^{2}}{\omega_{1} \omega_{2} \omega_{3}} \\
& +\mathcal{O}(\log \omega) .
\end{aligned}
$$

The series expansion parameter is given by $\omega$ which is the common scaling factor of $\omega_{I}$ 's in the Cardy limit, i.e., $\left|\omega_{I}\right| \sim \omega \ll 1$. Here $\mathfrak{a}, \mathfrak{b}, \mathfrak{c}$ and $\mathfrak{d}$ are the 't Hooft anomaly coefficients defined as follows,

$$
P_{8}=\frac{1}{4 !}\left(\mathfrak{a} \cdot c_{2}(R)^{2}+\mathfrak{b} \cdot c_{2}(R) p_{1}(T)+\mathfrak{c} \cdot p_{1}(T)^{2}+\mathfrak{d} \cdot p_{2}(T)\right)
$$

where $P_{8}$ is the anomaly 8 -form of the $6 \mathrm{~d}$ SCFT. Here, $c_{2}(R)$ is the second Chern class of SU(2) R-symmetry and $p_{1,2}(T)$ are the Pontryagin classes of the tangent bundle. Our Cardy formula (1.4) takes the form of the 'Cardy series' in the sense that it determines the free energy up to the subleading $\mathcal{O}\left(\omega^{-1}\right)$ order. If we perform Legendre transformation of the free energy, we obtain the asymptotic entropy in the Cardy limit. We found that the real part of the entropy is non-negative as long as the 't Hooft anomaly coefficients satisfy the following bound,

$$
\mathfrak{a}-4 \mathfrak{b}+16 \mathfrak{c} \geq 0
$$

which is satisfied for all examples analyzed in this paper. The same form of the inequality (1.6) was also found from the Renyi entropy of 6d SCFTs [43].

For 6d $(2,0)$ SCFT of ADE type, our Cardy formula determines the free energy as follows,

$$
\begin{aligned}
\log I= & -\frac{h_{G}^{\vee} d_{G}+r_{G}}{384} \frac{\left(\Delta_{R}^{2}-\Delta_{L}^{2}\right)^{2}}{\omega_{1} \omega_{2} \omega_{3}} \\
& -r_{G}\left[\frac{\left(\Delta_{R}^{2}+4 \pi^{2}\right)\left(\Delta_{L}^{2}+4 \pi^{2}\right)}{192 \omega_{1} \omega_{2} \omega_{3}}-\frac{\Delta_{R}^{2}+\Delta_{L}^{2}-8 \pi^{2}}{192} \frac{\omega_{1}^{2}+\omega_{2}^{2}+\omega_{3}^{2}}{\omega_{1} \omega_{2} \omega_{3}}\right]+\mathcal{O}(\log \omega)
\end{aligned}
$$

where $\Delta_{L, R}$ are chemical potentials for $\mathrm{SU}(2)_{L} \times \mathrm{SU}(2)_{R} \subset \mathrm{SO}(5)$ R-symmetry, and chemical potentials are constrained by $\Delta_{R}-\omega_{1}-\omega_{2}-\omega_{3}=2 \pi i$. Here, $\omega$ denotes a common scale for $\omega_{1,2,3}$ which is a small parameter. The group theoretic constants $h_{G}^{\vee}, d_{G}$ and $r_{G}$ are listed in table 1. In the large $N$ limit of $A_{N}$ type SCFT, the free energy (1.7) shows explicit $N^{3}$ growth which accounts for the entropy of BPS black holes in $A d S_{7} \times S^{4}$ [20, 26]. We make the similar analysis for the $D_{N}$ type theory in the large $N$ limit and found the correspondence with the entropy of BPS black holes in $A d S_{7} \times S^{4} / \mathbb{Z}_{2}$. 
The supersymmetric Casimir energy takes the same form with the equivariant integral of the anomaly polynomial [16]. For the Cardy free energy, we found that it can be obtained from the equivariant integral of the 'thermal anomaly polynomial' as follows,

$$
\log I=-\int P_{8}^{T}+\mathcal{O}(\log \omega)
$$

Here, $P_{8}^{T}$ is the thermal anomaly polynomial of the $6 \mathrm{~d}$ SCFT defined from the following replacement rule [42],

$$
P_{8}^{T}=P_{8}\left(p_{k}(T) \rightarrow p_{k}(T)-\frac{\mathbf{F}_{T}^{2}}{4 \pi^{2}} p_{k-1}(T)\right)
$$

where $\mathbf{F}_{T}$ is a field strength of the fictitious gauge field. The detailed explanation will be given in section 2 .

The rest of the paper is organized as follows. In section 2, we review the thermal derivative expansion of a thermal partition function. We introduce a formula derived in [42] that determines Chern-Simons terms in the effective action after the circle reduction. We also derive the Cardy formula (1.4) from the anomaly polynomial (1.5). In section 3, we compute the Cardy free energies of general $6 \mathrm{~d}(2,0)$ theories and some specific examples of $(1,0)$ theories with flavor symmetries. For some free 6d SCFTs, our Cardy formulas are explicitly checked by comparing with the superconformal indices. In section 4, we find that the Cardy free energy is given by the equivariant integral of the thermal anomaly polynomial. In section 5, we compute the entropy from the Cardy free energy. We show that the large $N$ free energies of $(2,0)$ SCFTs exactly account for the entropy of BPS black holes in the dual $A d S_{7}$. Also, we compute the asymptotic entropy in the Cardy limit and derive the bound (1.6).

\section{Thermal derivative expansion}

In this section, we review the derivative expansion of the thermal partition function of the even-dimensional QFT. We consider a QFT on $\mathcal{M}^{d}=M^{d-1} \times S^{1}$ where $d=2 n$. $M^{d-1}$ is $d$-1-dimensional compact manifold with $\partial M^{d-1}=0$ and $S^{1}$ is the Euclidean temporal circle which is fibered over $M^{d-1}$. We will assume that the QFT has a global symmetry $F$. The partition function takes the following definition,

$$
Z\left(\beta, \omega_{I}, \Delta_{i}\right)=\operatorname{Tr}\left[e^{-\beta E} \cdot \prod_{I=1}^{r_{M}} e^{-\omega_{I} J_{I}} \cdot \prod_{i=1}^{r_{F}} e^{-\Delta_{i} Q_{i}}\right]
$$

where $E$ is the energy, $J_{I}$ 's are the angular momenta of $M^{d-1}$, and $Q_{i}$ 's are the Cartan charges of the global symmetry $F$. The partition function depends on the chemical potentials $\beta, \omega_{I}$, and $\Delta_{i}$. The index $i$ runs from 1 to $r_{F}$ which is the rank of $F$, and $I$ runs from 1 to $r_{M}$ which is a rank of the isometry of $M^{d-1}$. $\beta$ is the circumference of $S^{1}$. 
The thermal partition function can be computed from the path integral of dynamical fields. The chemical potentials are encoded in the background fields $g_{\mu \nu}$ and $A_{\mu}$. The background metric $g_{\mu \nu}$ of $\mathcal{M}^{d}$ depends on $\omega_{I}$ 's, and the background gauge field $A_{\mu}$ depends on $\Delta_{i}$ 's. After integrating out all the dynamical fields, the partition function can be written as

$$
Z=\exp \left[-W\left(g_{\mu \nu}, A_{\mu}\right)\right]
$$

where $W$ is the effective action of the background fields.

However, it is impossible to evaluate the effective action in a generic setting since the path integral cannot be performed exactly. Instead, it is helpful to consider the small circle limit $\beta \ll \ell$ where $\ell$ is a curvature radius of $M^{d-1}$. In this limit, the dynamical fields can be reduced on $M^{d-1}$ into zero-modes with zero $S^{1}$ momentum and the Kaluza-Klein modes with non-zero $S^{1}$ momentum. The KK modes have the mass proportional to $\beta^{-1}$, and the path integral can be effectively evaluated as Gaussian integral. The zero-mode contribution to the effective action is subtle since they are light degrees on $M^{d-1}$. However, in section 2.2, we will introduce a certain scaling limit that the zero-mode contributions are suppressed.

One can canonically decompose the background fields on $\mathcal{M}^{d}$ into $S^{1}$ part and $M^{d-1}$ part. For the metric $g_{\mu \nu}$, it takes the following decomposition,

$$
g_{\mu \nu} d x^{\mu} d x^{\nu}=e^{-2 \Phi(x)}(d \tau+\mathbf{a})+h_{i j} d x^{i} d x^{j}, \quad \tau \sim \tau+\beta, \quad(1 \leq i, j \leq d-1)
$$

where $\Phi$ is a dilaton field, $\mathbf{a}=a_{i} d x^{i}$ is a graviphoton 1-form, and $h_{i j}$ is a metric of $M^{d-1}$. The background gauge field $\mathbf{A}=A_{i} d x^{i}$ and the affine connection $\boldsymbol{\Gamma}=\left(\Gamma^{\mu}{ }_{\nu}\right)_{\rho} d x^{\rho}$ are also decomposed as

$$
\mathbf{A}=A_{0}(d \tau+\mathbf{a})+\hat{\mathbf{A}}, \quad \boldsymbol{\Gamma}=\Gamma_{0}(d \tau+\mathbf{a})+\hat{\boldsymbol{\Gamma}}
$$

where $A_{0}, \Gamma_{0}$ are temporal components of $\mathbf{A}, \boldsymbol{\Gamma}$. Note that 1 -forms $\mathbf{a}, \hat{\mathbf{A}}$, and $\hat{\boldsymbol{\Gamma}}$ do not have a temporal component.

The effective action $W$ in (2.2) depends on the metric $h_{i j}$, 1-forms a, $\hat{\mathbf{A}}, \hat{\boldsymbol{\Gamma}}$, and scalars $A_{0}, \Gamma_{0}, \Phi$. Integrating out the $\mathrm{KK}$ modes yields the contribution to the effective action arranged as the form of an infinite series of the derivative expansion, which is the perturbative series of small $\beta$. Due to the presence of the zero-modes, effective action can also have non-perturbative terms of small $\beta$. The small $\beta$ limit of the partition function is of great importance in our case since it is related to the 'index point' where the partition function turns into the index [26]. In section 2.1, we will determine Chern-Simons terms in the effective action from the 't Hooft anomaly by following the works of Jensen, Loganayagam, and Yarom [40-42]. In section 2.2, we will explain the index limit of the partition function and show that the Cardy free energy can be determined from the Chern-Simons action only.

\subsection{Chern-Simons action and 't Hooft anomaly}

The 't Hooft anomaly is the anomaly of global symmetries induced by chiral fields in even dimensions [44]. The naive path integral yields the effective action of the background fields which suffers from the following 't Hooft anomaly,

$$
\delta W_{\mathrm{cons}}=-2 \pi i \int_{\mathcal{M}^{d}} I_{d}\left[g_{\mu \nu}, A_{\mu}\right]
$$


where $\delta$ collectively denotes the background gauge and the diffeomorphism transformation. We will call the effective action in (2.5) as the 'consistent action' since currents obtained from $W_{\text {cons }}$ satisfy the Wess-Zumino consistency condition [45]. The $d$-form anomaly $I_{d}$ is determined from the $d+2$-form anomaly polynomial $P_{d+2}$ through the following anomaly descent mechanism,

$$
d I_{d}=\delta I_{d+1}^{\mathrm{CS}}, \quad d I_{d+1}^{\mathrm{CS}}=P_{d+2}
$$

where $I_{d+1}^{\mathrm{CS}}$ is a Chern-Simons (CS) $d+1$-form made of $\mathbf{A}$ and $\boldsymbol{\Gamma}$. See [46] for a detailed review of the anomalies.

In this subsection, we focus on the CS terms in the effective action. In $W_{\text {cons }}$, there are two types of the CS actions: the gauge/diffeomorphism invariant CS action $W_{\text {ICS }}$ and the non-invariant CS action $W_{\text {NCS }}$. $W_{\text {ICS }}$ consists CS forms made of 1-forms $\hat{\mathbf{a}}, \hat{\mathbf{A}}$ and $\hat{\boldsymbol{\Gamma}}$ with properly quantized coefficients, and therefore they are invariant under the background gauge/diffeomorphism transformation. On the other hand, $W_{\text {NCS }}$ takes the form of normal CS terms but multiplied with the scalars $A_{0}, \Gamma_{0}$ and therefore it is the only gauge/diffeomorphism non-invariant part in the effective action. As a result, $W_{\mathrm{NCS}}$ is directly determined from the 't Hooft anomaly (2.5) as follows [42],

$$
W_{\mathrm{NCS}}=-2 \pi i \int_{\mathcal{M}^{d}} \frac{d t+\mathbf{a}}{d \mathbf{a}}\left[I_{d+1}^{\mathrm{CS}}-\hat{I}_{d+1}^{\mathrm{CS}}\right]
$$

The hatted form $\hat{I}_{d+1}^{\mathrm{CS}}$ is defined from $I_{d+1}^{\mathrm{CS}}$ by the following replacement,

$$
\hat{I}_{d+1}^{\mathrm{CS}}=I_{d+1}^{\mathrm{CS}}(\mathbf{A} \rightarrow \hat{\mathbf{A}}, \boldsymbol{\Gamma} \rightarrow \hat{\boldsymbol{\Gamma}}) .
$$

Throughout this paper, we will define the hatted notation as replacing 1-forms to their hatted forms as (2.8). The two-form $d \mathbf{a}$ in the denominator means the following operation,

$$
\frac{1}{d \mathbf{a}} \sum_{k=1} c_{k}(d \mathbf{a})^{k}=\sum_{k=1} c_{k}(d \mathbf{a})^{k-1}
$$

which is well-defined since $(d t+\mathbf{a})\left(I_{d+1}^{\mathrm{CS}}-\hat{I}_{d+1}^{\mathrm{CS}}\right)$ can be written as a finite power series of $d \mathbf{a}$ which starts from $(d \mathbf{a})^{1}$. Then it is straightforward to check that the variation of $W_{\mathrm{NCS}}$ generates the 't Hooft anomaly of the consistent action (2.5) as follows,

$$
\delta W_{\mathrm{NCS}}=-2 \pi i \int_{\mathcal{M}^{d}} \frac{d t+\mathbf{a}}{d \mathbf{a}}\left[d I_{d}-d \hat{I}_{d}\right]=-2 \pi i \int_{\mathcal{M}^{d}}\left[I_{d}-\hat{I}_{d}\right]=-2 \pi i \int_{\mathcal{M}^{d}} I_{d} .
$$

At the second equality of (2.10), we used that $\partial \mathcal{M}^{d}=0$ and $d \frac{d t+\mathbf{a}}{d \mathbf{a}}=1$. Although $\frac{d t+\mathbf{a}}{d \mathbf{a}}$ is not a properly defined differential form, it can be regarded as an -1 -form and $d \frac{d t+\mathbf{a}}{d \mathbf{a}}=1$ means the following property,

$$
d\left[\frac{d t+\mathbf{a}}{d \mathbf{a}} \sum_{k=1} c_{k}(d \mathbf{a})^{k}\right]=\sum_{k=1} c_{k}(d \mathbf{a})^{k}-\frac{d t+\mathbf{a}}{d \mathbf{a}} d\left[\sum_{k=1} c_{k}(d \mathbf{a})^{k}\right] .
$$

At the third equality of (2.10), we used that $\hat{I}_{d}$ does not contain the $d \tau$ component and vanish when integrating over $S^{1}$. 
By varying the consistent effective action with respect to the background fields, we obtain the consistent current $J_{\text {cons }}^{\mu}=\frac{\delta W_{\text {cons }}}{\delta A_{\mu}}$ and the consistent energy-momentum tensor $T_{\text {cons }}^{\mu \nu}=\frac{\delta W_{\text {cons }}}{\delta g_{\mu \nu}}$. However, they do not have the covariant form under the gauge/diffeomorphism transformation. This non-covariance can be cured by adding the local terms to $J_{\text {cons }}^{\mu}$ and $T_{\text {cons }}^{\mu \nu}$ which are called the Bardeen-Zumino polynomials [47]. The covariant current and the energy-momentum tensor can be obtained by varying the 'covariant action' $W_{\text {cov }}$ which is defined as follows,

$$
W_{\mathrm{cov}}=W_{\mathrm{cons}}+W_{\mathrm{bulk}}, \quad W_{\mathrm{bulk}}=2 \pi i \int_{\mathcal{N}^{d+1}} I_{d+1}^{\mathrm{CS}}
$$

where $\mathcal{N}^{d+1}$ is the bulk $d+1$-dimensional manifold whose boundary is given by $\mathcal{M}^{d}$. More precisely, we set $\mathcal{N}^{d+1}=S^{1} \times \mathcal{N}^{d}$ where $\partial N^{d}=M^{d-1}$. In the covariant action, $W_{\mathrm{NCS}}+W_{\text {bulk }}$ can be written as the following expression [42],

$$
W_{\mathrm{NCS}}+W_{\mathrm{bulk}}=2 \pi i \int_{\mathcal{N}^{d+1}} \frac{d t+\mathbf{a}}{d \mathbf{a}}\left[P_{d+2}-\hat{P}_{d+2}\right]
$$

which can be proven from the following identity,

$$
\frac{d t+\mathbf{a}}{d \mathbf{a}}\left(P_{d+1}^{\mathrm{CS}}-\hat{P}_{d+1}^{\mathrm{CS}}\right)=\left(I_{d+1}^{\mathrm{CS}}-\hat{I}_{d+1}^{\mathrm{CS}}\right)-d\left[\frac{d t+\mathbf{a}}{d \mathbf{a}}\left(I_{d+1}^{\mathrm{CS}}-\hat{I}_{d+1}^{\mathrm{CS}}\right)\right] .
$$

After integrating over $S^{1}$, the contribution from $\hat{I}_{d+1}^{\mathrm{CS}}$ vanishes due to its absence of the temporal component, and we obtain the desired result (2.13).

Until now, we have determined $W_{\text {NCS }}$ and $W_{\text {bulk }}$ which are not invariant under the background gauge/diffeomorphism transformation. However, the invariant CS action $W_{\text {ICS }}$ is not anomalous under the background gauge/diffeomorphism transformation. As a result, $W_{\text {ICS }}$ is not directly related to the anomalous transformation of the effective action (2.5). However, in [40-42], it was pointed out that one can impose another consistency condition called a 'consistency with the Euclidean vacuum' to determine $W_{\text {ICS }}$. Here, we introduce the condition and briefly sketch the derivation presented in [42].

Let us consider a $d$-dimensional manifold $\mathcal{M}^{d}=\mathbb{R}^{2, *} \times M^{d-2}$ where $\mathbb{R}^{2, *}$ is the twodimensional Euclidean plane with the origin removed. The plane $\mathbb{R}^{2, *}$ is fibered over the $d$-2-dimensional manifold $M^{d-2}$, and the fibration depends on the graviphoton $\mathbf{a}$. The metric of $\mathbb{R}^{2, *}$ is given by $d s_{\mathbb{R}^{2, *}}^{2}=r^{2} d \theta^{2}+d r^{2}$ with $\theta \sim \theta+2 \pi$ periodicity. We formally interpret $\theta$ as the Euclidean time $\tau$ by identifying $\tau=\frac{\beta}{2 \pi} \theta$, thus giving the metric $d s_{\mathbb{R}^{2, *}}^{2}=$ $\frac{4 \pi^{2} r^{2}}{\beta^{2}} d \tau^{2}+d r^{2}$. The total metric of $\mathcal{M}^{d}$ is then given by follows,

$$
d s^{2}=\frac{4 \pi^{2} r^{2}}{\beta^{2}}(d \tau+\mathbf{a})^{2}+d r^{2}+d s_{M^{d-2}}^{2} .
$$

Now, we turn on the specific background field configuration of $\mathbf{a}$ and $\hat{\mathbf{A}}$ which are non-zero only on $M^{d-2}$. Let us consider an infinitesimal metric perturbation on $\mathbb{R}^{2, *}$ as $\delta g_{\tau r} d \tau d r$. The consistency with the Euclidean vacuum requires the following condition,

$$
T_{\mathrm{cov}}^{\tau r}=\frac{\partial W_{\mathrm{cov}}}{\delta g_{\tau r}}=0
$$


Since the graviphoton a and the background gauge field $\hat{\mathbf{A}}$ are only turned on $M^{d-2}$, the partition function should reproduce correlation functions of the Euclidean vacuum on $\mathbb{R}^{2, *}$. As a result, the rotational invariance of the Euclidean vacuum is equivalent to the consistency condition (2.16). In this geometric setting, it should be noted that the derivative expansion of $W_{\text {cov }}$ is generally ineffective since the angular circle of $\mathbb{R}^{2, *}$ becomes large if we go far from the origin. In this case, the infinite series of the derivative expansion has to be resummed, as well as non-perturbative terms, in order to compute the effective action or the generic correlation functions. However, in [42], it was argued that one can construct certain background field configurations such that only finite number of terms in $W_{\text {bulk }}, W_{\mathrm{NCS}}$, and $W_{\mathrm{ICS}}$ contribute to $T_{\text {cov }}^{\tau r}$ among infinitely many terms in the effective action. In such cases, $T^{\tau r}$ can be exactly computed regardless of the validity of the derivative expansion. As a result, the consistency condition (2.16) can be used to determine $W_{\text {ICS }}$ since $W_{\text {bulk }}$ and $W_{\text {NCS }}$ are already determined as (2.13).

As a conclusion, together with the results shown in (2.7) and (2.12), the three types of CS actions in $W_{\text {cov }}$ take the following expression,

$$
\begin{aligned}
& W_{\text {bulk }}=2 \pi i \int_{\mathcal{N}^{d+1}} I_{d+1}^{\mathrm{CS}} \\
& W_{\mathrm{NCS}}=-2 \pi i \int_{\mathcal{M}^{d}} \frac{d t+\mathbf{a}}{d \mathbf{a}}\left[I_{d+1}^{\mathrm{CS}}-\hat{I}_{d+1}^{\mathrm{CS}}\right] \\
& W_{\mathrm{ICS}}=-2 \pi i \int_{\mathcal{M}^{d}} \frac{d t+\mathbf{a}}{d \mathbf{a}}\left[\Delta I_{d+1}^{\mathrm{CS}}-\Delta \hat{I}_{d+1}^{\mathrm{CS}}\right]_{\mathbf{A}^{T}=0} .
\end{aligned}
$$

Here, $\Delta I_{d+1}^{\mathrm{CS}}$ is another CS $d+1$-form which will be explained shortly, and $\mathbf{A}^{T}$ is a fictitious $\mathrm{U}(1)$ gauge field defined as follows,

$$
\mathbf{A}^{T}=\frac{2 \pi i}{\beta}(d \tau+\mathbf{a})+\hat{\mathbf{A}}^{T}
$$

Using $\mathbf{A}^{T}$, we define a 'thermal anomaly polynomial' from the ordinary anomaly polynomial by the following replacement rule [42],

$$
P_{d+2}^{T}=P_{d+2}\left(p_{k}(T) \rightarrow p_{k}(T)-\frac{\mathbf{F}_{T}^{2}}{4 \pi^{2}} p_{k-1}(T)\right)
$$

where $p_{k}(T)$ is the $k^{\prime}$ th Pontryagin class of the tangent bundle. Then, $\Delta I_{d+1}^{\mathrm{CS}}$ is defined as

$$
P_{d+2}^{T}=d I_{d+1}^{T} \quad \text { where } \quad I_{d+1}^{T}=I_{d+1}^{\mathrm{CS}}+\Delta I_{d+1}^{\mathrm{CS}} .
$$

Note that the fictitious field $\mathbf{A}^{T}$ will be turned off at the end of the computation, but its effect on $\Delta \hat{I}_{d+1}^{\mathrm{CS}}$ survives since $\hat{\mathbf{A}}^{T}$ is non-zero. The sum of the three actions in (2.17) can be written as the following simple form,

$$
W_{\mathrm{bulk}}+W_{\mathrm{NCS}}+W_{\mathrm{ICS}}=2 \pi i \int_{\mathcal{N}^{d+1}} \frac{d t+\mathbf{a}}{d \mathbf{a}}\left[P_{d+2}^{T}-\hat{P}_{d+2}^{T}\right]_{\mathbf{A}^{T}=0} .
$$

The dimensional reduction of (2.21) gives the CS forms on $M^{d-1}$ which are the only CS terms in $W_{\text {cov }}$. Therefore, all CS terms in the covariant action can be computed from the anomaly polynomial of the theory. 
For the quantized charges, the chemical potentials in (2.1) have the periodicity along the imaginary direction, and their periodic shift is related to the large gauge transformation on $S^{1}$. If we properly regulate the partition function to preserve the periodicity of the chemical potentials, it is natural to obtain the covariant action as the effective action in (2.2). The reason is that the covariant action itself is invariant under the background gauge/diffeomorphism transformation even when the 't Hooft anomaly is non-zero. Therefore, in the rest of this paper, we will use $W_{\text {cov }}$ to compute the partition function defined in (2.1) and (2.2) to ensure the periodicity of the chemical potentials. As a related matter, the presence of the Casimir energy prefactor can break the periodicity of the chemical potential. Therefore, we speculate that the covariant action is related to the spectral/state-counting part of the superconformal index. In section 3, it will be shown that our Cardy formula gives the Cardy free energy of the superconformal index without the Casimir energy prefactor.

Aside from the CS action, the effective action receives two other types of contributions, which we will denote $W_{\text {non-CS }}$ and $W_{\text {zero }} . W_{\text {non-CS }}$ is the non-CS terms in the derivative expansion, and $W_{\text {zero }}$ is the terms that are not captured in the derivative expansion due to the contribution of the dynamical zero-modes. As a result, the partition function (2.1) can be written as

$$
Z=\exp \left[-W_{\mathrm{CS}}-W_{\text {non-CS }}-W_{\text {zero }}\right]
$$

where $W_{\mathrm{CS}} \equiv W_{\mathrm{ICS}}+W_{\mathrm{NCS}}+W_{\text {bulk }}$ is the CS action determined in (2.21). Without evaluating $W_{\text {non-CS }}$ and $W_{\text {zero }}$, the partition function can not be precisely obtained. However, in the following subsection, we will show that in the certain scaling limit where the partition function turns into the index, the evaluation of $W_{\mathrm{CS}}$ is sufficient to determine the behavior of the index in the Cardy limit.

\subsection{6d index from Chern-Simons action}

In this section, we focus on $6 \mathrm{~d}$ superconformal field theories with $\mathrm{SU}(2)_{R}$ R-symmetry on $S^{5} \times S^{1}$. We will show that the modified index can be obtained from the small $S^{1}$ limit of the partition function. If we further take the Cardy limit $\left(\left|\omega_{I}\right| \ll 1\right)$ after taking the small $S^{1}$ limit, the leading term and a few subleading terms of the free energy can be determined from the CS action on $S^{5}$.

The $6 \mathrm{~d}$ modified superconformal index (1.2) counts BPS states on $S^{5}$, and takes the following definition,

$$
I(\Delta, \omega)=\operatorname{Tr}\left[e^{-\beta\left\{\mathcal{Q}, \mathcal{Q}^{\dagger}\right\}} \cdot e^{-\Delta_{R} Q_{R}} \cdot e^{-\omega_{1} J_{1}-\omega_{2} J_{2}-\omega_{3} J_{3}}\right]
$$

where $\mathcal{Q}$ is the defining supercharge of the index. Let us take $\mathcal{Q}$ such that $\left\{\mathcal{Q}, \mathcal{Q}^{\dagger}\right\}=$ $E-r^{-1}\left(4 Q_{R}+J_{1}+J_{2}+J_{3}\right)$ where $r$ is the radius of $S^{5}$. The chemical potentials are constrained by $\Delta_{R}-\omega_{1}-\omega_{2}-\omega_{3}=2 \pi i$ to preserve the supersymmetry. We turned off other flavor symmetry chemical potentials for simplicity. See section 3 for the detailed explanation. The modified index counts the BPS states that saturate the BPS bound $\left\{\mathcal{Q}, \mathcal{Q}^{\dagger}\right\} \geq 0$, and therefore, the index does not depend on $\beta$. After explicitly inserting 
$\left\{\mathcal{Q}, \mathcal{Q}^{\dagger}\right\}$ factor, one can rewrite the index as follows,

$$
I(\Delta, \omega)=\operatorname{Tr}\left[e^{-\beta E} \cdot e^{-\left(\Delta_{R}-4 \frac{\beta}{r}\right) Q_{R}} \cdot e^{-\left(\omega_{1}-\frac{\beta}{r}\right) J_{1}-\left(\omega_{2}-\frac{\beta}{r}\right) J_{2}-\left(\omega_{3}-\frac{\beta}{r}\right) J_{3}}\right] .
$$

The partition function of the same $6 \mathrm{~d}$ theory takes the following definition,

$$
Z(\beta, \Delta, \omega)=\operatorname{Tr}\left[e^{-\beta E} \cdot e^{-\Delta_{R} Q_{R}} \cdot e^{-\omega_{1} J_{1}-\omega_{2} J_{2}-\omega_{3} J_{3}}\right] .
$$

For the partition function, the chemical potentials are all independent, and it counts all states on $S^{5}$. It is straightforward to see that the index (2.24) lies on a special point of the thermal partition function given by $\Delta_{R}-\omega_{1}-\omega_{2}-\omega_{3}=2 \pi i-\frac{\beta}{r}$. By using the fact that the index does not depend on $\beta$, one can simply take $\beta \rightarrow 0$ limit which turns out to be beneficial for the background field analysis. Then, the index $I$ can be obtained from the partition function $Z$ in the following limit,

$$
I(\Delta, \omega)=\lim _{\beta / r \rightarrow 0} Z(\beta, \Delta, \omega) \quad \text { at } \quad \Delta_{R}-\omega_{1}-\omega_{2}-\omega_{3}=2 \pi i .
$$

The original Cardy formula of $2 \mathrm{~d}$ CFT [32] counts the degeneracy of the states in a large spatial momentum limit, which is called the Cardy limit. The higher dimensional generalization of the Cardy limit can be understood as the limit where $J_{I}$ 's are large. In the canonical ensemble, such a limit can be obtained by taking $\left|\omega_{I}\right| \ll 1$ [26]. The Cardy limit $\left|\omega_{I}\right| \ll 1$ of the index $I$ can be studied from the partition function $Z$ by taking the $\beta \ll r$ limit first, and then taking $\left|\omega_{I}\right| \ll 1$ limit later. It can be accomplished by considering the following scaling limit,

$$
\frac{\beta}{r} \ll\left|\omega_{1,2,3}\right| \ll 1
$$

Now, let us evaluate the various terms of the effective action in the above scaling limit (2.27).

With the chemical potentials turned on as (2.25), the background metric on $S^{5} \times S^{1}$ is given as follows,

$$
\begin{aligned}
g_{\mu \nu} d x^{\mu} d x^{\nu} & =r^{2}\left[d \theta_{1}^{2}+\sin ^{2} \theta_{1} d \theta_{2}^{2}+\sum_{i=1}^{3} n_{i}^{2}\left(d \phi_{i}-\frac{i \omega_{i}}{\beta} d \tau\right)^{2}\right]+d \tau^{2} \\
n_{1} & =\cos \theta_{1}, \quad n_{2}=\sin \theta_{1} \cos \theta_{2}, \quad n_{3}=\sin \theta_{1} \sin \theta_{2}
\end{aligned}
$$

with temporal periodicity $\tau \sim \tau+\beta$. The background $\mathrm{SU}(2)_{R}$ R-symmetry gauge field $\mathbf{A}_{\mathrm{SU}(2)}$ is turned on as

$$
\mathbf{A}_{\mathrm{SU}(2)}=\mathbf{A} \cdot T_{\mathrm{SU}(2)}, \quad \mathbf{A}=\frac{\Delta_{R}}{\beta} d \tau
$$

where $T_{\mathrm{SU}(2)}$ is the Cartan generator of $\mathrm{SU}(2)$ normalized as $\operatorname{tr}_{\mathbf{a d j}}\left[T_{\mathrm{SU}(2)}^{2}\right]=2$, and $\mathbf{A}$ is the $\mathrm{U}(1)$ Cartan gauge field. One may worry that due to $\beta$ in the denominators of the background fields, the effective action might be singular in our scaling limit. However, we will show that effective action is well-defined in our scaling limit. A similar setting was considered in [26]. 
As introduced in (2.3), the metric and the gauge fields are reduced on $S^{5}$ as follows,

$$
\begin{aligned}
g_{\mu \nu} d x^{\nu} d x^{\nu} & =e^{-2 \Phi}(d \tau+\mathbf{a})^{2}+h_{i j} d x^{i} d x^{j} \\
h_{i j} d x^{i} d x^{j} & =r^{2}\left(d \theta_{1}^{2}+\sin ^{2} \theta_{1} d \theta_{2}^{2}+n_{i}^{2} d \phi_{i}^{2}+\frac{r^{2}}{\beta^{2}} \frac{\left(\sum_{i} \omega_{i} n_{i}^{2} d \phi_{i}\right)^{2}}{1-\frac{r^{2}}{\beta^{2}} \sum_{i} n_{i}^{2} \omega_{i}^{2}}\right)
\end{aligned}
$$

where $h_{i j}$ is a metric of the squashed $S^{5}$. We will temporarily assume that $\omega_{i}$ 's are purely imaginary so that the metric (2.30) is well defined. The extension to the complex $\omega_{i}$ 's can be understood as the analytic continuation. The dilaton $\Phi$ and the graviphoton a are

$$
e^{-2 \Phi}=1-\frac{r^{2}}{\beta^{2}} \sum_{i} n_{i}^{2} \omega_{i}^{2}, \quad \mathbf{a}=-i \frac{r^{2} \sum_{i} \omega_{i} n_{i}^{2} d \phi_{i}}{\beta\left(1-\frac{r^{2}}{\beta^{2}} \sum_{i} n_{i}^{2} \omega_{i}^{2}\right)}
$$

The background gauge field and the affine connection are

$$
\mathbf{A}=A_{0}(d \tau+\mathbf{a})+\hat{\mathbf{A}}, \quad \boldsymbol{\Gamma}=\Gamma_{0}(d \tau+\mathbf{a})+\hat{\boldsymbol{\Gamma}}
$$

We should keep in mind that, due to (2.29), we should plug $A_{0}=\frac{\Delta_{R}}{\beta}$ and $\hat{\mathbf{A}}=-A_{0} \mathbf{a}$ after the computation. Now, let us evaluate the three contributions $W_{\mathrm{CS}}, W_{\text {non-CS}}$, and $W_{\text {zero }}$ in the effective action (2.22) one by one.

First, we consider the CS terms $W_{\mathrm{CS}}$ on $S^{5}$. The explicit structure of the CS action is determined from the thermal anomaly polynomial as (2.21). Let us consider the most general form of the $6 \mathrm{~d}$ anomaly polynomial with $\mathrm{SU}(2) \mathrm{R}$-symmetry and the tangent bundle as follows,

$$
P_{8}=\frac{1}{4 !}\left(\mathfrak{a} \cdot c_{2}(R)^{2}+\mathfrak{b} \cdot c_{2}(R) p_{1}(T)+\mathfrak{c} \cdot p_{1}(T)^{2}+\mathfrak{d} \cdot p_{2}(T)\right)
$$

where $\mathfrak{a}, \mathfrak{b}, \mathfrak{c}$ and $\mathfrak{d}$ are the 't Hooft anomaly coefficients. The second Chern class of SU(2) R-symmetry is defined as

$$
c_{2}(R)=\frac{1}{4(2 \pi)^{2}} \operatorname{Tr}\left[\mathbf{F}_{\mathrm{SU}(2)}^{2}\right]=\frac{1}{4(2 \pi)^{2}} \mathbf{F}^{2}
$$

where the capital trace is defined as $\operatorname{Tr} \equiv\left(h_{G}^{\vee}\right)^{-1} \operatorname{tr}_{\mathbf{a d j}}$ and $\mathbf{F}=d \mathbf{A}$. Note that the dual Coxeter number of SU(2) is $h_{G}^{\vee}=2$. The first and the second Pontryagin classes of the tangent bundle are

$$
p_{1}(T)=-\frac{1}{2(2 \pi)^{2}} \operatorname{tr}\left[\mathbf{R}^{2}\right], \quad p_{2}(T)=\frac{1}{(2 \pi)^{4}}\left(-\frac{1}{4} \operatorname{tr}\left[\mathbf{R}^{4}\right]+\frac{1}{8}\left(\operatorname{tr}\left[\mathbf{R}^{2}\right]\right)^{2}\right)
$$

where $\mathbf{R}$ is a curvature two-form of $6 \mathrm{~d}$ manifold. After applying the replacement rule (2.19), the thermal anomaly polynomial is

$$
P_{8}^{T}=\frac{1}{4 !}\left[\mathfrak{a} \cdot c_{2}(R)^{2}+\mathfrak{b} \cdot c_{2}(R)\left(p_{1}(T)-\frac{\mathbf{F}_{T}^{2}}{4 \pi^{2}}\right)+\mathfrak{c} \cdot\left(p_{1}(T)-\frac{\mathbf{F}_{T}^{2}}{4 \pi^{2}}\right)^{2}+\mathfrak{d} \cdot\left(p_{2}(T)-\frac{\mathbf{F}_{T}^{2}}{4 \pi^{2}} p_{1}(T)\right)\right]
$$


where $\mathbf{F}_{T}$ is the field strength of a fictitious $\mathrm{U}(1)$ gauge field defined in (2.18). The thermal anomaly polynomial can be rearranged as follows,

$$
\begin{aligned}
& P_{8}^{T}=\frac{\mathfrak{a}}{6144 \pi^{4}} \mathbf{F}^{4}-\frac{\mathfrak{b}}{1536 \pi^{4}} \mathbf{F}^{2} \mathbf{F}_{T}^{2}+\frac{\mathfrak{c}}{384 \pi^{4}} \mathbf{F}_{T}^{4} \quad \rightarrow \quad \text { gauge anomaly } \\
& -\frac{\mathfrak{b}}{3072 \pi^{4}} \operatorname{tr}\left[\mathbf{R}^{2}\right] \mathbf{F}^{2}+\frac{2 \mathfrak{c}+\mathfrak{d}}{768 \pi^{4}} \operatorname{tr}\left[\mathbf{R}^{2}\right] \mathbf{F}_{T}^{2} \quad \rightarrow \quad \text { mixed anomaly } \\
& -\frac{\mathfrak{d}}{1536 \pi^{4}} \operatorname{tr}\left[\mathbf{R}^{4}\right]+\frac{2 \mathfrak{c}+\mathfrak{d}}{3072 \pi^{4}} \operatorname{tr}\left[\mathbf{R}^{2}\right]^{2} \quad \rightarrow \quad \text { gravitational anomaly. }
\end{aligned}
$$

In (2.37), we divide the anomalies into the three types: gauge, mixed, and gravitational anomalies. The CS action is then given by

$$
W_{\mathrm{CS}}=2 \pi i \int_{\mathcal{N}^{7}} \frac{d \tau+\mathbf{a}}{d \mathbf{a}}\left[P_{8}^{T}-\hat{P}_{8}^{T}\right]_{\mathbf{A}^{T}=0}
$$

where $\mathcal{N}^{7}=B^{6} \times S^{1}$ with $\partial B^{6}=S^{5}$ such as a six-dimensional solid ball. The two-forms can be written in terms of hatted two-forms as follows.

$$
\begin{aligned}
\mathbf{F} & =d \mathbf{A}=\hat{\mathbf{F}}+A_{0} d \mathbf{a}+\mathcal{O}(d \tau+\mathbf{a}) \\
\mathbf{R} & =d \boldsymbol{\Gamma}+\boldsymbol{\Gamma}^{2}=\hat{\mathbf{R}}+\Gamma_{0} d \mathbf{a}+\mathcal{O}(d \tau+\mathbf{a}) \\
\mathbf{F}_{T} & =\hat{\mathbf{F}}_{T}+\frac{2 \pi i}{\beta} d \mathbf{a} .
\end{aligned}
$$

Then, the thermal anomaly polynomial $P_{8}^{T}$ can be written as

$$
P_{8}^{T}=P_{8}^{T}\left(\mathbf{F} \rightarrow \hat{\mathbf{F}}+A_{0} d \mathbf{a}, \mathbf{R} \rightarrow \hat{\mathbf{R}}+\Gamma_{0} d \mathbf{a}, \mathbf{F}_{T} \rightarrow \hat{\mathbf{F}}_{T}+\frac{2 \pi i}{\beta} d \mathbf{a}\right)+\mathcal{O}(d \tau+\mathbf{a}) .
$$

The terms of order $\mathcal{O}(d \tau+\mathbf{a})$ do not contribute to the CS action since they vanish when evaluating (2.38).

Now, let us compute the CS action from the thermal anomaly polynomial (2.37). We first consider the gauge anomaly which gives the gauge CS action as follows,

$$
\begin{aligned}
W_{\text {gauge-CS }}=2 \pi i \int_{\mathcal{N}^{7}} \frac{d \tau+\mathbf{a}}{d \mathbf{a}}[ & \frac{\mathfrak{a}}{6144 \pi^{4}}\left(\left(\hat{\mathbf{F}}+A_{0} d \mathbf{a}\right)^{4}-\hat{\mathbf{F}}^{4}\right) \\
& -\frac{\mathfrak{b}}{1536 \pi^{4}}\left(\left(\hat{\mathbf{F}}+A_{0} d \mathbf{a}\right)^{2}\left(\hat{\mathbf{F}}_{T}+\frac{2 \pi i}{\beta} d \mathbf{a}\right)^{2}-\hat{\mathbf{F}}^{2} \hat{\mathbf{F}}_{T}^{2}\right) \\
& \left.+\frac{\mathfrak{c}}{384 \pi^{4}}\left(\left(\hat{\mathbf{F}}_{T}+\frac{2 \pi i}{\beta} d \mathbf{a}\right)^{4}\right)-\hat{\mathbf{F}}_{T}^{4}\right]_{\mathbf{A}_{T}=0} .
\end{aligned}
$$

The integral is defined on the $\mathcal{N}^{7}=S^{1} \times B^{6}$, but it can be reduced on the boundary since $A_{0}$ is a constant. After integrating over $S^{1}$, we obtain,

$$
\begin{aligned}
W_{\text {gauge-CS }}=2 \pi i \beta \int_{S^{5}} & {\left[\frac{\mathfrak{a}}{6144 \pi^{4}}\left(A_{0}^{4} \mathbf{a} d \mathbf{a} d \mathbf{a}+4 A_{0}^{3} \hat{\mathbf{A}} d \mathbf{a} d \mathbf{a}+6 A_{0}^{2} \mathbf{a} d \hat{\mathbf{A}} d \hat{\mathbf{A}}+4 A_{0} \hat{\mathbf{A}} d \hat{\mathbf{A}} d \hat{\mathbf{A}}\right)\right.} \\
& \left.-\frac{\mathfrak{b}}{384 \pi^{2} \beta^{2}} \mathbf{a} d \hat{\mathbf{A}} d \hat{\mathbf{A}}-\frac{\mathfrak{c}}{24 \pi^{2} \beta^{4}} \mathbf{a} d \mathbf{a} d \mathbf{a}\right] \\
= & 2 \pi i \int_{S^{5}}\left(-\frac{\mathfrak{a}}{6144 \pi^{4}}\left(\beta A_{0}\right)^{4}-\frac{\mathfrak{b}}{384 \pi^{2}}\left(\beta A_{0}\right)^{2}-\frac{\mathfrak{c}}{24}\right) \cdot \frac{1}{\beta^{3}} \mathbf{a} d \mathbf{a} d \mathbf{a}
\end{aligned}
$$


where we used $\hat{\mathbf{A}}=-A_{0}$ a in the second equality. By a similar way, we can obtain the mixed CS action as follows,

$$
W_{\text {mixed-CS }}=2 \pi i \int_{S^{5}}\left(\frac{\mathfrak{b}}{3072 \pi^{4}}\left(\beta A_{0}\right)^{2}+\frac{2 \mathfrak{c}+\mathfrak{d}}{192 \pi^{2}}\right) \cdot \frac{1}{\beta} \mathbf{a t r}\left[\hat{\mathbf{R}}^{2}\right]
$$

The gravitational CS action is much more complicated, and it is unclear whether its form is uniquely fixed on $S^{5}$. The main complication is that $\Gamma_{0}$ is not a constant unlike to $A_{0}$. Here, we list a few sample terms that can appear in the gravitational CS action as follows,

$$
W_{\text {grav-CS }} \supset \beta \int_{S^{5}} \operatorname{tr}\left[\Gamma_{0}^{4}\right] \mathbf{a} d \mathbf{a} d \mathbf{a}, \quad \beta \int_{S^{5}} \operatorname{tr}\left[\Gamma_{0}^{2}\right]^{2} \mathbf{a} d \mathbf{a} d \mathbf{a}, \ldots
$$

where many other complicated terms are omitted. However, we do not need to evaluate $W_{\text {gravi-CS }}$ since they are subleading in our scaling limit, as will be discussed in the next paragraph.

In our scaling limit, those three types of CS terms have different scaling behavior. The gauge CS terms scales of order $\mathcal{O}\left(\left(\frac{\beta}{r}\right)^{0} \omega^{-3}\right)$, the mixed CS terms scales of order $\mathcal{O}\left(\left(\frac{\beta}{r}\right)^{0} \omega^{-1}\right)$, and the gravitational CS terms scales of order $\mathcal{O}\left(\left(\frac{\beta}{r}\right)^{0} \omega \log \omega\right)$. For the gauge CS action, the corresponding CS term gives

$$
\frac{1}{\beta^{3}} \int_{S^{5}} \mathbf{a} d \mathbf{a} d \mathbf{a}=i \frac{(2 \pi)^{3} \omega_{1} \omega_{2} \omega_{3}}{\left(\omega_{1}^{2}-\frac{\beta^{2}}{r^{2}}\right)\left(\omega_{2}^{2}-\frac{\beta^{2}}{r^{2}}\right)\left(\omega_{3}^{2}-\frac{\beta^{2}}{r^{2}}\right)}=\frac{8 i \pi^{3}}{\omega_{1} \omega_{2} \omega_{3}}\left[1+\mathcal{O}\left(\left(\frac{\beta}{r \omega}\right)^{2}\right)\right] .
$$

For the mixed CS action, the corresponding CS term gives

$$
\frac{1}{\beta} \int_{S^{5}} \mathbf{a} \operatorname{tr}\left[\hat{\mathbf{R}}^{2}\right]=2 i \frac{(2 \pi)^{3} \omega_{1} \omega_{2} \omega_{3}\left(\omega_{1}^{2}+\omega_{2}^{2}+\omega_{3}^{2}\right)}{\left(\omega_{1}^{2}-\frac{\beta^{2}}{r^{2}}\right)\left(\omega_{2}^{2}-\frac{\beta^{2}}{r^{2}}\right)\left(\omega_{3}^{2}-\frac{\beta^{2}}{r^{2}}\right)}=\frac{16 i \pi^{3}\left(\omega_{1}^{2}+\omega_{2}^{2}+\omega_{3}^{2}\right)}{\omega_{1} \omega_{2} \omega_{3}}\left[1+\mathcal{O}\left(\left(\frac{\beta}{r \omega}\right)^{2}\right)\right] .
$$

For the gravitational CS action, we present a contribution from a single term as an example as follows,

$$
\begin{aligned}
& \beta \int_{S^{5}} \operatorname{tr}\left[\Gamma_{0}^{4}\right] \mathbf{a} d \mathbf{a} d \mathbf{a}=32 i \pi^{3}\left(\frac{\omega_{1}^{4}+\omega_{2}^{4}+\omega_{3}^{4}}{2 \omega_{1} \omega_{2} \omega_{3}}+\frac{2 \omega_{1} \omega_{2} \omega_{3}\left(3 \omega_{1}^{2}+\omega_{2}^{2}+\omega_{3}^{2}\right)}{\left(\omega_{1}^{2}-\omega_{2}^{2}\right)\left(\omega_{1}^{2}-\omega_{3}^{2}\right)} \log \omega_{1}\right. \\
& \left.+\frac{2 \omega_{1} \omega_{2} \omega_{3}\left(\omega_{1}^{2}+3 \omega_{2}^{2}+\omega_{3}^{2}\right)}{\left(\omega_{2}^{2}-\omega_{1}^{2}\right)\left(\omega_{2}^{2}-\omega_{3}^{2}\right)} \log \omega_{2}+\frac{2 \omega_{1} \omega_{2} \omega_{3}\left(\omega_{1}^{2}+\omega_{2}^{2}+3 \omega_{3}^{2}\right)}{\left(\omega_{3}^{2}-\omega_{1}^{2}\right)\left(\omega_{3}^{2}-\omega_{2}^{2}\right)} \log \omega_{3}\right) \times\left[1+\mathcal{O}\left(\left(\frac{\beta}{r \omega}\right)^{2}\right)\right] .
\end{aligned}
$$

In the constant dilaton background where $\omega \equiv \omega_{1,2,3}$, we checked that the other gravitational CS terms are also of order $\mathcal{O}(\omega \log \omega)$ or lower, which are subleading than the other CS terms. Moreover, as we will argue shortly, the zero-mode contribution $W_{\text {zero }}$ is considered to be of order $\mathcal{O}(\log \omega)$ which is more dominant than the gravitational CS terms. Since the zero-mode contribution cannot be determined in the anomaly-based approach, we will ignore the gravitational CS terms, and keep the only gauge and mixed CS terms to compute the Cardy free energy. From (2.45) and (2.46), we obtain the following results 
for the anomaly polynomial given in (2.33),

$$
\begin{aligned}
W_{\text {gauge-CS }} & =\left(\frac{\mathfrak{a}}{384} \Delta_{R}^{4}+\frac{\mathfrak{b} \pi^{2}}{24} \Delta_{R}^{2}+\frac{2 \mathfrak{c} \pi^{4}}{3}\right) \frac{1}{\omega_{1} \omega_{2} \omega_{3}}+\mathcal{O}\left(\left(\frac{\beta}{r \omega}\right)^{2}\right) \\
W_{\text {mixed-CS }} & =-\left(\frac{\mathfrak{b}}{96} \Delta_{R}^{2}+\frac{(2 \mathfrak{c}+\mathfrak{d}) \pi^{2}}{6}\right) \frac{\omega_{1}^{2}+\omega_{2}^{2}+\omega_{3}^{2}}{\omega_{1} \omega_{2} \omega_{3}}+\mathcal{O}\left(\left(\frac{\beta}{r \omega}\right)^{2}\right) .
\end{aligned}
$$

Now, let us discuss the non-CS terms in the derivative expansion. In order to simplify our discussion, we shall consider the constant dilaton background $\omega \equiv \omega_{1,2,3}$ when evaluating non-CS terms. The generic non-CS Lagrangian can be constructed from the graviphoton field strength $f_{i j}=\beta^{-1}\left(\partial_{i} a_{j}-\partial_{j} a_{i}\right)$, the gauge field strength $F_{i j}=\partial_{i} \hat{A}_{j}-\partial_{j} \hat{A}_{i}$, the curvature $\hat{R}_{j k l}{ }_{j k l}$, and the two temporal scalars $\beta A_{0}, \beta \Gamma_{j 0}^{i}$. The exceeding number of the lower indices should be contracted with the metric $\left(\beta e^{-\Phi}\right)^{2} h^{i j}$. Also, there is an overall metric determinant factor $\sqrt{\operatorname{det}\left[\left(\beta^{-1} e^{\Phi}\right)^{2} h_{i j}\right]}$. Note that all the quantities are normalized to be dimensionless. In our scaling limit, those background fields are the order of ${ }^{1}$

$$
\begin{aligned}
& f_{i j} \sim \mathcal{O}\left(\left(\frac{\beta}{r}\right)^{0} \omega^{-1}\right), \quad F_{i j} \sim \Delta \cdot \mathcal{O}\left(\left(\frac{\beta}{r}\right)^{0} \omega^{-1}\right), \quad \hat{R}_{j k l}^{i} \sim \mathcal{O}\left(\left(\frac{\beta}{r}\right)^{0} \omega^{0}\right), \\
& \beta A_{0} \sim \Delta \cdot \mathcal{O}\left(\left(\frac{\beta}{r}\right)^{0} \omega^{0}\right), \quad \beta \Gamma_{j 0}^{i} \sim \mathcal{O}\left(\left(\frac{\beta}{r}\right)^{0} \omega^{1}\right), \quad\left(\beta e^{-\Phi}\right)^{2} h^{i j} \sim \mathcal{O}\left(\left(\frac{\beta}{r}\right)^{0} \omega^{2}\right), \\
&\left(\beta e^{-\Phi}\right)^{-5} \sqrt{\operatorname{det}\left[h_{i j}\right]} \sim \mathcal{O}\left(\left(\frac{\beta}{r}\right)^{1} \omega^{-6}\right)
\end{aligned}
$$

Now let us consider a general form of the Lagrangian $\mathcal{L}\left(n_{1}, n_{2}, n_{3}, n_{4}, n_{5}, n_{6}\right)$ which consists of $n_{1}$ numbers of $f_{i j}, n_{2}$ numbers of $F_{i j}, n_{3}$ numbers of $\hat{R}_{j k l}^{i}, n_{4}$ numbers of $\beta A_{0}, n_{5}$ numbers of $\beta \Gamma^{i}{ }_{j 0}$, and $2 n_{6}$ numbers of derivative $\nabla_{i}$ where all $n_{i}$ 's are non-negative integers. The indices are contracted with $n_{1}+n_{2}+n_{3}+n_{6}$ numbers of $\left(\beta e^{-\Phi}\right)^{2} h^{i j}$. After integrating over $S^{5}$, the lowest possible order of the action is

$$
\int_{S^{5}} \mathcal{L}\left(n_{1}, n_{2}, n_{3}, n_{4}, n_{5}, n_{6}\right) \sim \Delta^{n_{2}+n_{4}} \cdot \mathcal{O}\left(\left(\frac{\beta}{r}\right)^{1} \cdot \omega^{-6+n_{1}+n_{2}+2 n_{3}+n_{5}+2 n_{6}}\right)
$$

which is suppressed in our scaling limit due to the overall $(\beta / r)^{1}$ factor. Our result $(2.50)$ does not follow a naive derivative expansion argument that the $n$ 'th derivative term comes with an additional $\beta^{n}$ power, since the background fields themselves consists $\beta^{-1}$ factor. However, in our scaling limit, all non-CS terms are suppressed by the overall $\beta$ factor, and higher derivative terms are more suppressed with higher $\omega$ powers. Therefore, the thermal derivative expansion is valid for our background fields.

The result (2.50) is based on the leading order counting of (2.49). An honest evaluation of the action can give a smaller leading order if the cancellation happens at the expected leading order. In any case, the true leading order should be equal or subleading than the

\footnotetext{
${ }^{1}\left(\beta e^{-\Phi}\right)^{2} h^{i j}$ has non-zero components of order $\mathcal{O}\left(\left(\frac{\beta}{r}\right)^{-2} \omega^{4}\right)$ for $x^{i, j} \in\left\{\phi^{1}, \phi^{2}, \phi^{3}\right\}$. However, those components are contracted with $\partial / \partial \phi^{1,2,3}$, and their contribution to the action vanishes since the background fields are independent of $\phi^{1,2,3}$ coordinates.
} 
predicted leading order in (2.50). For all non-CS terms on $S^{5}$ evaluated in [26], we checked that they are consistent with our prediction.

Lastly, let us discuss $W_{\text {zero }}$, which is the contribution from the dynamical zero-modes. The circle compactification of generic $6 \mathrm{~d}$ SCFT yields 5 d gauge theory with $\mathcal{N}=1$ or 2 supersymmetry, and the structure of the zero-modes is given by $5 \mathrm{~d}$ gauge theory on the squashed $S^{5}$. The $5 \mathrm{~d}$ theory has a gauge coupling constant $g_{\mathrm{YM}}^{2} \sim \beta e^{-\Phi}$ which becomes $g_{\mathrm{YM}}^{2} \sim \mathcal{O}(r \omega)$ in our scaling limit. The $5 \mathrm{~d}$ SYM is weakly coupled in the IR, and it is unlikely that the free energy has the terms proportional to the negative power of the coupling constant. Possibly the most severe IR divergence of the free energy would be $\log g_{\mathrm{YM}}^{2} \sim$ $\mathcal{O}(\log \omega)[26]$. In this paper, we assume that $W_{\text {zero }} \sim \mathcal{O}(\log \omega)$ in our scaling limit.

As a conclusion, we would like to emphasize that both $W_{\text {non-CS }}$ and $W_{\text {zero }}$ are suppressed in our scaling limit, but with different origins. $W_{\text {non-CS }}$ is of order $\mathcal{O}\left(\frac{\beta}{r}\right)$, and it does not contribute to the index at the strict index point $\beta / r \rightarrow 0$ given in (2.26). On the other hand, $W_{\text {zero }}$ can contribute to the index, but it is subleading in the Cardy limit $|\omega| \ll 1$. As a result, Cardy free energy can be obtained from the CS action as follows,

$$
\log I=-\lim _{\beta / r \rightarrow 0} W_{\mathrm{CS}}+\mathcal{O}(\log \omega) .
$$

For the anomaly polynomial given in (2.33), we obtain the following results,

$$
\begin{aligned}
\log I= & -\left(\frac{\mathfrak{a}}{384} \Delta_{R}^{4}+\frac{\mathfrak{b} \pi^{2}}{24} \Delta_{R}^{2}+\frac{2 \mathfrak{c} \pi^{4}}{3}\right) \frac{1}{\omega_{1} \omega_{2} \omega_{3}}+\left(\frac{\mathfrak{b}}{96} \Delta_{R}^{2}+\frac{(2 \mathfrak{c}+\mathfrak{d}) \pi^{2}}{6}\right) \frac{\omega_{1}^{2}+\omega_{2}^{2}+\omega_{3}^{2}}{\omega_{1} \omega_{2} \omega_{3}} \\
& +\mathcal{O}(\log \omega) .
\end{aligned}
$$

where the chemical potentials are constrained by $\Delta_{R}-\omega_{1}-\omega_{2}-\omega_{3}=2 \pi i$. The 't Hooft anomaly determines not only the leading order $\mathcal{O}\left(\omega^{-3}\right)$ term, but also the subleading corrections up to $\mathcal{O}\left(\omega^{-1}\right)$. The $\mathcal{O}(\log \omega)$ correction includes the zero-mode contribution, which cannot be determined from the anomaly.

Before moving on to our main results, let us make a few comments on the role of the supersymmetry in our derivation of (2.52). First of all, one might ask that the background fields and the effective action in this section are not supersymmetric. This is because we considered the on-shell action where the background fields are fixed from the chemical potentials of the modified index. The full off-shell multiplets of the $5 \mathrm{~d}$ supergravity have much more field contents, including fermionic superpartners [39]. The off-shell multiplets boil down to the bosonic fields such as graviton, graviphoton, and dilaton on the on-shell point. Here, we constructed the on-shell action made of those fields.

However, supersymmetry still plays a subtle role here. When computing the superconformal index (2.23), we use the fact that the index does not depend on $\beta$ and take the $\beta \rightarrow 0$ limit. Such a limit is crucial for our background field analysis since all the non-CS terms can be suppressed. Therefore, by exploiting the $\beta$-independence of the superconformal index, one can obtain the Cardy free energy of the superconformal index from the background CS terms only.

In the following sections, we will compute the Cardy formula of various 6d SCFTs with $(1,0)$ and $(2,0)$ supersymmetries using the method explained so far. 


\section{Cardy formulas of 6d SCFTs}

As a preliminary, let us define the superconformal index and the modified index of $6 \mathrm{~d}$ SCFTs and elaborate on the relation between them.

The superconformal index of $6 \mathrm{~d}(2,0)$ SCFT on $S^{5} \times \mathbb{R}_{\text {time }}$ is defined as follows,

$$
\mathcal{I}\left(\Delta_{1,2}, \omega_{1,2,3}\right)=\operatorname{Tr}\left[(-1)^{F} \cdot e^{-\beta\left\{\mathcal{Q}, \mathcal{Q}^{\dagger}\right\}} \cdot e^{-\Delta_{1} Q_{1}-\Delta_{2} Q_{2}} \cdot e^{-\omega_{1} J_{1}-\omega_{2} J_{2}-\omega_{3} J_{3}}\right] .
$$

The superconformal algebra of $6 \mathrm{~d}(2,0)$ theories has a maximal bosonic subalgebra $\mathrm{SO}(6,2) \times \mathrm{SO}(5)$ [48]. The Cartans of $\mathrm{SO}(6,2)$ are the energy $E$ and three angular momenta $J_{1,2,3}$. The $(2,0)$ R-symmetry is $\mathrm{SO}(5) \supset \mathrm{SU}(2)_{L} \times \mathrm{SU}(2)_{R}$ whose Cartans are $Q_{L, R}$. In (3.1), $Q_{1,2}$ are defined as $Q_{1}=Q_{R}+Q_{L}$ and $Q_{2}=Q_{R}-Q_{L}$. Sixteen Poincare supercharges of $(2,0)$ theories are labelled by $\mathcal{Q}_{t_{1}, t_{2}}^{s_{1}, s_{2}, s_{3}}$ where $s_{I}, t_{I}= \pm 1$ and $s_{1} s_{2} s_{3}<0$. The anticommutation relation between the supercharges is given as follows,

$$
\left\{\mathcal{Q}_{t_{1}, t_{2}}^{s_{1}, s_{2}, s_{3}},\left(\mathcal{Q}_{t_{1}, t_{2}}^{s_{1}, s_{2}, s_{3}}\right)^{\dagger}\right\}=E+r^{-1}\left(s_{1} J_{1}+s_{2} J_{2}+s_{3} J_{3}-2\left(t_{1} Q_{1}+t_{2} Q_{2}\right)\right)
$$

where $r$ is the radius of $S^{5}$. When the operators in the trace of (3.1) are acted on the supercharge, we obtain the following factor,

$$
\begin{aligned}
& e^{-\omega_{1} J_{1}-\omega_{2} J_{2}-\omega_{3} J_{3}} \cdot e^{-\Delta_{1} Q_{1}-\Delta_{2} Q_{2}} \cdot \mathcal{Q}_{t_{1}, t_{2}}^{s_{1}, s_{2}, s_{3}} \\
& \quad=e^{\frac{1}{2}\left(s_{1} \omega_{1}+s_{2} \omega_{2}+s_{3} \omega_{3}+t_{1} \Delta_{1}+t_{2} \Delta_{2}\right)} \cdot \mathcal{Q}_{t_{1}, t_{2}}^{s_{1}, s_{2}, s_{3}} \cdot e^{-\omega_{1} J_{1}-\omega_{2} J_{2}-\omega_{3} J_{3}} \cdot e^{-\Delta_{1} Q_{1}-\Delta_{2} Q_{2}} .
\end{aligned}
$$

Let us define the superconformal index (3.1) with the supercharge $\mathcal{Q} \equiv \mathcal{Q}_{++}^{---}$. Then, the BPS states saturate the BPS bound given by

$$
E \geq r^{-1}\left(J_{1}+J_{2}+J_{3}+4 Q_{R}\right) .
$$

For the supercharge $\mathcal{Q}$ to commute with the operators in the trace, we should impose the following condition for the chemical potentials,

$$
\Delta_{R}-\omega_{1}-\omega_{2}-\omega_{3}=0 \quad(\bmod 4 \pi i)
$$

where $\Delta_{R}=\Delta_{1}+\Delta_{2}$ and $\Delta_{L}=\Delta_{1}-\Delta_{2}$. As a result, the index is independent of $\beta$ and we can turn it off to zero.

Now, we introduce the modified version of the $(2,0)$ index defined as follows,

$$
I\left(\Delta_{L, R}, \omega_{1,2,3}\right)=\operatorname{Tr}\left[e^{-\omega_{1} J_{1}-\omega_{2} J_{2}-\omega_{3} J_{3}} \cdot e^{-\Delta_{R} Q_{R}-\Delta_{L} Q_{L}}\right]
$$

where the chemical potentials are constrained by $\Delta_{R}-\omega_{1}-\omega_{2}-\omega_{3}=2 \pi i$. Although the original index $\mathcal{I}$ (3.1) and the modified index $I$ (3.6) are different in their expressions, they are equivalent up to the shift of the chemical potentials as follows,

$$
\mathcal{I}\left(\Delta_{L}, \Delta_{R}, \omega_{1}, \omega_{2}, \omega_{3}\right)=I\left(\Delta_{L}, \Delta_{R}, \omega_{1}, \omega_{2}, \omega_{3}-2 \pi i\right) .
$$

The $2 \pi i$ shift of $\omega_{3}$ in (3.6) yields $e^{2 \pi i J_{3}}$ factor. Since $J_{3}$ is quantized to be integer for bosonic states and half-integer for fermionic states, one can replace $e^{2 \pi i J_{3}}$ by $(-1)^{F}$, thus proving (3.7). 
Now, let us move on to the indices of $(1,0)$ SCFT. The superconformal index of $6 \mathrm{~d}$ $(1,0)$ SCFT is defined as follows,

$$
\mathcal{I}\left(\Delta, \omega_{1,2,3}, x_{i}\right)=\operatorname{Tr}\left[(-1)^{F} \cdot e^{-\beta\left\{\mathcal{Q}, \mathcal{Q}^{\dagger}\right\}} \cdot e^{-\Delta_{R} Q_{R}} \cdot e^{-\omega_{1} J_{1}-\omega_{2} J_{2}-\omega_{3} J_{3}} \cdot \prod_{i} e^{-m_{i} F_{i}}\right] .
$$

The superconformal algebra of $6 \mathrm{~d}(1,0)$ theories has maximal bosonic subgroup $\mathrm{SO}(6,2) \times$ $\mathrm{SU}(2)_{R}$ [48]. The Cartans of $\mathrm{SO}(6,2)$ are the energy $E$ and three angular momenta $J_{1,2,3}$. The Cartan of $\mathrm{SU}(2)_{R}$ R-symmetry is $Q_{R}$. For $(1,0)$ theories, there can be flavor symmetries with Cartan charges $F_{i}$ and chemical potentials $m_{i}$. Eight Poincare supercharges of $(1,0)$ theories are labelled by $\mathcal{Q}_{t}^{s_{1}, s_{2}, s_{3}}$ with $s_{I}, t= \pm 1$ with $s_{1} s_{2} s_{3}<0$. We take $\mathcal{Q} \equiv \mathcal{Q}_{+}^{--}$to define the index. Then the BPS bound is given by

$$
E \geq r^{-1}\left(J_{1}+J_{2}+J_{3}+4 Q_{R}\right)
$$

with the following chemical potential constraints,

$$
\Delta_{R}-\omega_{1}-\omega_{2}-\omega_{3}=0 \quad(\bmod 4 \pi i)
$$

Now, we introduce the modified version of the $(1,0)$ index defined as follows,

$$
I=\operatorname{Tr}\left[e^{-\Delta_{R} Q_{R}} \cdot e^{-\omega_{1} J_{1}-\omega_{2} J_{2}-\omega_{3} J_{3}} \cdot \prod_{i} e^{-m_{i} F_{i}}\right]
$$

where the chemical potentials are constrained by $\Delta_{R}-\omega_{1}-\omega_{2}-\omega_{3}=2 \pi i$. As well as the $(2,0)$ index, the modified index and the original index of $(1,0)$ SCFTs related as follows,

$$
\mathcal{I}\left(m_{i}, \Delta_{R}, \omega_{1}, \omega_{2}, \omega_{3}\right)=I\left(m_{i}, \Delta_{R}, \omega_{1}, \omega_{2}, \omega_{3}-2 \pi i\right)
$$

\section{$3.1(1,0)$ SCFT without flavor chemical potentials}

In this section, we consider a modified superconformal index of general $6 \mathrm{~d}(1,0)$ given as follows,

$$
I=\operatorname{Tr}\left[e^{-\Delta_{R} Q_{R}} \cdot e^{-\omega_{1} J_{1}-\omega_{2} J_{2}-\omega_{3} J_{3}}\right]
$$

where chemical potentials are constrained by $\Delta_{R}-\omega_{1}-\omega_{2}-\omega_{3}=2 \pi i$. The anomaly polynomial of the $6 \mathrm{~d}$ SCFT takes the following form,

$$
P_{8}=\frac{1}{4 !}\left(\mathfrak{a} \cdot c_{2}(R)^{2}+\mathfrak{b} \cdot c_{2}(R) p_{1}(T)+\mathfrak{c} \cdot p_{1}(T)^{2}+\mathfrak{d} \cdot p_{2}(T)\right)
$$

with the theory dependent coefficients $\mathfrak{a}, \mathfrak{b}, \mathfrak{c}$ and $\mathfrak{d}$. The definitions of $c_{2}(R)$ and $p_{1,2}(T)$ are given in (2.34) and (2.35). From the above anomaly polynomial, we derived the following Cardy formula in section 2.2 as follows,

$$
\log I=-\left(\frac{\mathfrak{a}}{384} \Delta_{R}^{4}+\frac{\mathfrak{b} \pi^{2}}{24} \Delta_{R}^{2}+\frac{2 \mathfrak{c} \pi^{4}}{3}\right) \frac{1}{\omega_{1} \omega_{2} \omega_{3}}+\left(\frac{\mathfrak{b}}{96} \Delta_{R}^{2}+\frac{(2 \mathfrak{c}+\mathfrak{d}) \pi^{2}}{6}\right) \frac{\omega_{1}^{2}+\omega_{2}^{2}+\omega_{3}^{2}}{\omega_{1} \omega_{2} \omega_{3}}+\mathcal{O}(\log \omega)
$$


By inserting the chemical potential constraint $\Delta_{R}=2 \pi i+\omega_{1}+\omega_{2}+\omega_{3}$, we can rearrange the Cardy formula (3.15) as follows,

$$
\begin{aligned}
\log I= & -\frac{\pi^{4}(\mathfrak{a}-4 \mathfrak{b}+16 \mathfrak{c})}{24} \frac{1}{\omega_{1} \omega_{2} \omega_{3}}+\frac{i \pi^{3}(\mathfrak{a}-2 \mathfrak{b})}{12} \frac{\omega_{1}+\omega_{2}+\omega_{3}}{\omega_{1} \omega_{2} \omega_{3}} \\
& +\frac{\pi^{2}(3 \mathfrak{a}-4 \mathfrak{b}+16 \mathfrak{c}+8 \mathfrak{d})}{48} \frac{\omega_{1}^{2}+\omega_{2}^{2}+\omega_{3}^{2}}{\omega_{1} \omega_{2} \omega_{3}}+\frac{\pi^{2}(3 \mathfrak{a}-2 \mathfrak{b})}{24} \frac{\omega_{1} \omega_{2}+\omega_{2} \omega_{3}+\omega_{3} \omega_{1}}{\omega_{1} \omega_{2} \omega_{3}} \\
& +\mathcal{O}(\log \omega)
\end{aligned}
$$

In this section, we will test our Cardy formula by comparing it with the known indices of $6 \mathrm{~d}(1,0)$ free hyper and tensor multiplets. The indices are computed in the appendix B.

The modified superconformal indices of $(1,0)$ free hyper and tensor multiplets are given as follow,

$$
\begin{aligned}
I_{\text {hyp }} & =\exp \sum_{n=1}^{\infty} \frac{2}{n} \frac{\left(-q_{1} q_{2} q_{3}\right)^{n}}{\left(1-q_{1}^{2 n}\right)\left(1-q_{2}^{2 n}\right)\left(1-q_{3}^{2 n}\right)} \\
I_{\text {ten }} & =\exp \sum_{n=1}^{\infty} \frac{1}{n} \frac{\left(q_{1} q_{2} q_{3}\right)^{2 n}-\left(q_{1} q_{2}\right)^{2 n}-\left(q_{2} q_{3}\right)^{2 n}-\left(q_{3} q_{1}\right)^{2 n}}{\left(1-q_{1}^{2 n}\right)\left(1-q_{2}^{2 n}\right)\left(1-q_{3}^{2 n}\right)}
\end{aligned}
$$

where fugacities are defined as $q_{I}=e^{-\omega_{I} / 2}$. In the Cardy limit, the free energy can be expanded as follows,

$$
\begin{aligned}
& \log I_{\text {hyp }}=\sum_{n=1}^{\infty}\left[\frac{2(-1)^{n}}{n^{4}} \frac{1}{\omega_{1} \omega_{2} \omega_{3}}-\frac{(-1)^{n}}{12 n^{2}} \frac{\omega_{1}^{2}+\omega_{2}^{2}+\omega_{3}^{2}}{\omega_{1} \omega_{2} \omega_{3}}+\mathcal{O}(\omega)\right] \\
& \log I_{\text {ten }}=\sum_{n=1}^{\infty}\left[-\frac{2}{n^{4}} \frac{1}{\omega_{1} \omega_{2} \omega_{3}}-\frac{1}{6 n^{2}} \frac{\omega_{1}^{2}+\omega_{2}^{2}+\omega_{3}^{2}-3\left(\omega_{1} \omega_{2}+\omega_{2} \omega_{3}+\omega_{3} \omega_{1}\right)}{\omega_{1} \omega_{2} \omega_{3}}-\frac{1}{2 n}+\mathcal{O}(\omega)\right] .
\end{aligned}
$$

As can be seen, the sum over $n$ becomes divergent after $\mathcal{O}\left(\omega^{-1}\right)$ order. This divergence signals the breakdown of the perturbative expansion of small $\omega$ after $\mathcal{O}\left(\omega^{-1}\right)$. We expect that after $\mathcal{O}\left(\omega^{-1}\right)$ order, there exist $\mathcal{O}(\log \omega)$ terms that cannot be captured from the perturbative expansion. At least for the unrefined abelian $(2,0)$ tensor index, the existence of those terms will be explicitly shown in the next subsection. Therefore, after summing over $n$, we can expand our Cardy free energy as follows,

$$
\begin{aligned}
& \log I_{\text {hyp }}=-\frac{7 \pi^{4}}{360} \frac{1}{\omega_{1} \omega_{2} \omega_{3}}+\frac{\pi^{2}}{144} \frac{\omega_{1}^{2}+\omega_{2}^{2}+\omega_{3}^{2}}{\omega_{1} \omega_{2} \omega_{3}}+\mathcal{O}(\log \omega) \\
& \log I_{\text {ten }}=-\frac{\pi^{4}}{45} \frac{1}{\omega_{1} \omega_{2} \omega_{3}}-\frac{\pi^{2}}{36} \frac{\omega_{1}^{2}+\omega_{2}^{2}+\omega_{3}^{2}}{\omega_{1} \omega_{2} \omega_{3}}+\frac{\pi^{2}}{12} \frac{\omega_{1} \omega_{2}+\omega_{2} \omega_{3}+\omega_{3} \omega_{1}}{\omega_{1} \omega_{2} \omega_{3}}+\mathcal{O}(\log \omega) .
\end{aligned}
$$

Now, we compare the free energy (3.19) with our Cardy formula (3.16). The anomaly coefficients of the free hyper and tensor multiplets are given by [14]

$$
\begin{aligned}
\text { hyper: } & \mathfrak{a}=0, & \mathfrak{b}=0, & \mathfrak{c}=\frac{7}{240}, & \mathfrak{d}=-\frac{1}{60} \\
\text { tensor: } & \mathfrak{a}=1, & \mathfrak{b}=\frac{1}{2}, & \mathfrak{c}=\frac{23}{240}, & \mathfrak{d}=-\frac{29}{60}
\end{aligned}
$$

By plugging the anomaly coefficients (3.20) to the Cardy formula (3.16), we can check that our Cardy formula gives the correct answers for all the result given in (3.19). 


\section{$3.2 \quad(2,0)$ SCFT of ADE type}

$6 \mathrm{~d}(2,0)$ superconformal field theories have a well-known ADE classification $[49,50]$. They are engineered from IIB string theory on $\mathbb{C}^{2} / \Gamma$ where $\Gamma$ is a discrete ADE subgroup of $\mathrm{SU}(2)$. For $A_{N-1} \mathrm{SCFT}$ with a single free tensor multiplet, it has the M-theory origin as a worldvolume theory of $N$ coincident M5-branes [51]. In this section, we study the superconformal indices of $(2,0)$ theories in the Cardy limit using the background field method introduced in section 2 .

The background fields of $6 \mathrm{~d}(2,0)$ SCFTs are the metric $g_{\mu \nu}$ and the R-symmetry gauge field $\mathbf{A}_{\mathrm{SU}(2)}^{L, R}$. The explicit form of the metric and the graviphoton fields are written in (2.30) and (2.31). We turn on Cartans of two $\mathrm{SU}(2)$ gauge fields $\mathbf{A}_{\mathrm{SU}(2)}^{L, R}$ of $\mathrm{SU}(2)_{L} \times \mathrm{SU}(2)_{R} \subset$ $\mathrm{SO}(5)$ as follows,

$$
\mathbf{A}_{\mathrm{SU}(2)}^{L, R}=\mathbf{A}^{L, R} \cdot T_{\mathrm{SU}(2)}
$$

where $T_{\mathrm{SU}(2)}$ is the Cartan generator of $\mathrm{SU}(2)$ normalized as $\operatorname{tr}_{\mathbf{a d j}}\left[\left(T_{\mathrm{SU}(2)}\right)^{2}\right]=2 . \mathbf{A}^{L, R}$ are $\mathrm{U}(1)$ Cartan gauge fields that have the following form,

$$
\mathbf{A}^{L, R}=A_{0}^{L, R}(d \tau+\mathbf{a})+\hat{\mathbf{A}}^{L, R}, \quad A_{0}^{L, R}=\frac{\Delta_{L, R}}{\beta} .
$$

At the end of the computation, we will insert $\hat{\mathbf{A}}^{L, R}=-A_{0}^{L, R} \mathbf{a}$ to make the background gauge fields purely temporal.

As explained in section 2, the Chern-Simons terms of the background effective action is determined from the anomaly polynomial. The anomaly polynomial of a $6 \mathrm{~d}(2,0)$ SCFT of type $G$ is given by [14]

$$
P_{8}=\frac{h_{G}^{\vee} d_{G}}{24} p_{2}(N)+\frac{r_{G}}{48}\left[p_{2}(N)-p_{2}(T)+\frac{1}{4}\left(p_{1}(N)-p_{1}(T)\right)^{2}\right] .
$$

Here, $h_{G}^{\vee}$ is a dual Coxeter number, $d_{G}$ is a group dimension, and $r_{G}$ is a rank of $G$. Various group-theoretic constants are listed in the table 1. The Pontryagin classes of the tangent bundle are defined in (2.35). For SO(5) normal bundle, its Pontryagin classes can be written in terms of Chern-classes of $\mathrm{SU}(2)_{L, R}$ as follows,

$$
\begin{aligned}
p_{1}(N) & =-2\left(c_{2}(L)+c_{2}(R)\right), \quad p_{2}(N)=\left(c_{2}(L)-c_{2}(R)\right)^{2} \\
c_{2}(L, R) & =\frac{1}{4(2 \pi)^{2}} \operatorname{Tr}\left[\left(\mathbf{F}_{\mathrm{SU}(2)}^{L, R}\right)^{2}\right]
\end{aligned}
$$

where $\mathbf{F}_{\mathrm{SU}(2)}^{L, R}$ is a field strength two-form of $\mathbf{A}_{\mathrm{SU}(2)}^{L, R}$, and $c_{2}$ is the second Chern-class. The capital trace is defined as $\operatorname{Tr} \equiv\left(h_{G}^{\vee}\right)^{-1} \operatorname{tr}_{\mathbf{a d j}}$.

According to the replacement rule (2.19), the thermal anomaly polynomial $P_{8}^{T}$ is given as follows,

$$
P_{8}^{T}=\frac{h_{G}^{\vee} d_{G}}{24} p_{2}(N)+\frac{r_{G}}{48}\left[p_{2}(N)-\left(p_{2}(T)-\frac{\mathbf{F}_{T}^{2}}{4 \pi^{2}} p_{1}(T)\right)+\frac{1}{4}\left(p_{1}(N)-p_{1}(T)+\frac{\mathbf{F}_{T}^{2}}{4 \pi^{2}}\right)^{2}\right]
$$


where $\mathbf{F}_{T}$ is the field strength two-form of the fictitious gauge field $\mathbf{A}^{T}$ which was introduced in (2.18). The CS action on $S^{5}$ are given as (2.21),

$$
W_{\mathrm{CS}}=2 \pi i \int_{\mathcal{N}^{7}} \frac{d t+\mathbf{a}}{d \mathbf{a}}\left[P_{8}^{T}-\hat{P}_{8}^{T}\right]_{\mathbf{A}^{T}=0}
$$

where $\mathcal{N}^{7}=B^{6} \times S^{1}$ with a six-dimensional solid ball $B^{6}$ with the boundary $S^{5}$. After some computation, we obtain the following Chern-Simons action on $S^{5}$,

$$
\begin{aligned}
W_{\mathrm{CS}}= & \frac{i}{\beta^{3}} \int_{S^{5}} \mathbf{a} d \mathbf{a} d \mathbf{a} \cdot\left(-\frac{h_{G}^{\vee} d_{G}+r_{G}}{3072 \pi^{3}}\left(\Delta_{R}^{2}-\Delta_{L}^{2}\right)^{2}-\frac{r_{G}}{1536 \pi^{3}}\left(\Delta_{R}^{2}+4 \pi^{2}\right)\left(\Delta_{L}^{2}+4 \pi^{2}\right)\right) \\
& +\frac{i}{\beta} \int_{S^{5}} \mathbf{a t r}\left[\hat{\mathbf{R}}^{2}\right] \cdot \frac{r_{G}}{3072 \pi^{3}}\left(\Delta_{R}^{2}+\Delta_{L}^{2}-8 \pi^{2}\right)+W_{\text {grav-CS }}
\end{aligned}
$$

The gravitational CS action $W_{\text {grav-CS }}$ can be ignored in the Cardy limit. The integration over $S^{5}$ can be evaluated by (2.45) and (2.46). In the scaling limit (2.27), we obtain

$$
\begin{aligned}
W_{\mathrm{CS}}= & \frac{h_{G}^{\vee} d_{G}+r_{G}}{384} \frac{\left(\Delta_{R}^{2}-\Delta_{L}^{2}\right)^{2}}{\omega_{1} \omega_{2} \omega_{3}} \\
& +r_{G}\left[\frac{\left(\Delta_{R}^{2}+4 \pi^{2}\right)\left(\Delta_{L}^{2}+4 \pi^{2}\right)}{192 \omega_{1} \omega_{2} \omega_{3}}-\frac{\Delta_{R}^{2}+\Delta_{L}^{2}-8 \pi^{2}}{192} \frac{\omega_{1}^{2}+\omega_{2}^{2}+\omega_{3}^{2}}{\omega_{1} \omega_{2} \omega_{3}}\right]+W_{\text {grav-CS }}+\mathcal{O}\left(\frac{\beta}{r}\right)
\end{aligned}
$$

Then the Cardy free energy can be obtained from (3.28) and (2.51) as follows,

$$
\begin{aligned}
\log I= & -\frac{h_{G}^{\vee} d_{G}+r_{G}}{384} \frac{\left(\Delta_{R}^{2}-\Delta_{L}^{2}\right)^{2}}{\omega_{1} \omega_{2} \omega_{3}} \\
& -r_{G}\left[\frac{\left(\Delta_{R}^{2}+4 \pi^{2}\right)\left(\Delta_{L}^{2}+4 \pi^{2}\right)}{192 \omega_{1} \omega_{2} \omega_{3}}-\frac{\Delta_{R}^{2}+\Delta_{L}^{2}-8 \pi^{2}}{192} \frac{\omega_{1}^{2}+\omega_{2}^{2}+\omega_{3}^{2}}{\omega_{1} \omega_{2} \omega_{3}}\right]+\mathcal{O}(\log \omega)
\end{aligned}
$$

where the chemical potentials are constrained by $\Delta_{R}-\omega_{1}-\omega_{2}-\omega_{3}=2 \pi i$.

The chemical potentials are periodic variables with $4 \pi i$ periodicity. In the Cardy limit, $\Delta_{L}$ is the only relevant parameters with the periodicity since the other chemical potentials are fixed near $\omega_{1,2,3} \simeq 0$ and $\Delta_{R} \simeq 2 \pi i$. We will see in the following paragraphs that for $6 \mathrm{~d}(2,0)$ theories, our expression $(3.29)$ is valid in the following range which we shall call a 'canonical chamber',

$$
-2 \pi<\operatorname{Im}\left[\Delta_{L}\right]<2 \pi
$$

The index outside the canonical chamber can be obtained from (3.29) by $4 \pi i$ periodic shift of $\Delta_{L}$.

Aside from the interacting $6 \mathrm{~d}(2,0)$ SCFTs with ADE type, there is a free $6 \mathrm{~d}(2,0)$ theory, which is made of the Abelian $(2,0)$ tensor multiplet. For a single Abelian tensor multiplet, its anomaly polynomial is given as follows,

$$
P_{8}=\frac{1}{48}\left[p_{2}(N)-p_{2}(T)+\frac{1}{4}\left(p_{1}(N)-p_{1}(T)\right)^{2}\right] .
$$

The anomaly polynomial (3.31) takes the same form with (3.23) if we set $h_{G}^{\vee} d_{G}=0$ and $r_{G}=1$. Therefore, the Cardy free energy of the tensor multiplet is given as follows,

$$
\begin{aligned}
\log I= & -\frac{1}{384} \frac{\left(\Delta_{R}^{2}-\Delta_{L}^{2}\right)^{2}}{\omega_{1} \omega_{2} \omega_{3}} \\
& -\left[\frac{\left(\Delta_{R}^{2}+4 \pi^{2}\right)\left(\Delta_{L}^{2}+4 \pi^{2}\right)}{192 \omega_{1} \omega_{2} \omega_{3}}-\frac{\Delta_{R}^{2}+\Delta_{L}^{2}-8 \pi^{2}}{192} \frac{\omega_{1}^{2}+\omega_{2}^{2}+\omega_{3}^{2}}{\omega_{1} \omega_{2} \omega_{3}}\right]+\mathcal{O}(\log \omega) .
\end{aligned}
$$


The closed-form expression of the superconformal index of the free $(2,0)$ tensor multiplet is known, and we can directly check our Cardy formula (3.32). The superconformal index of the free tensor multiplet is known as follows,

$$
\begin{aligned}
& \mathcal{I}_{\mathrm{U}(1)}=\operatorname{Tr}\left[(-1)^{F} \cdot e^{-\Delta_{L} Q_{L}-\Delta_{R} Q_{R}} \cdot e^{-\omega_{1} J_{1}-\omega_{2} J_{2}-\omega_{3} J_{3}}\right], \quad\left(\Delta_{R}-\omega_{1}-\omega_{2}-\omega_{3}=0\right) \\
& =\exp \sum_{n=1}^{\infty} \frac{1}{n}\left[\frac{e^{-n \frac{\Delta_{R}+\Delta_{L}}{2}}+e^{-n \frac{\Delta_{R}-\Delta_{L}}{2}}-e^{-n\left(\omega_{1}+\omega_{2}\right)}-e^{-n\left(\omega_{2}+\omega_{3}\right)}-e^{-n\left(\omega_{3}+\omega_{1}\right)}+e^{-n\left(\omega_{1}+\omega_{2}+\omega_{3}\right)}}{\left(1-e^{-n \omega_{1}}\right)\left(1-e^{-n \omega_{2}}\right)\left(1-e^{-n \omega_{3}}\right)}\right] .
\end{aligned}
$$

As explained in (3.7), the superconformal index (3.33) can be turned into the modified index by shifting the chemical potentials as $\omega_{3} \rightarrow \omega_{3}+2 \pi i$. Then we obtain the following modified index,

$$
\begin{aligned}
& I_{\mathrm{U}(1)}=\operatorname{Tr}\left[e^{-\Delta_{R} Q_{R}-\Delta_{L} Q_{L}} \cdot e^{-\omega_{1} J_{1}-\omega_{2} J_{2}-\omega_{3} J_{3}}\right], \quad\left(\Delta_{R}-\omega_{1}-\omega_{2}-\omega_{3}=2 \pi i\right) \\
& =\exp \sum_{n=1}^{\infty} \frac{1}{n}\left[\frac{e^{-n \frac{\Delta_{R}+\Delta_{L}}{2}}+e^{-n \frac{\Delta_{R}-\Delta_{L}}{2}}-e^{-n\left(\omega_{1}+\omega_{2}\right)}-e^{-n\left(\omega_{2}+\omega_{3}\right)}-e^{-n\left(\omega_{3}+\omega_{1}\right)}+e^{-n\left(\omega_{1}+\omega_{2}+\omega_{3}\right)}}{\left(1-e^{-n \omega_{1}}\right)\left(1-e^{-n \omega_{2}}\right)\left(1-e^{-n \omega_{3}}\right)}\right] .
\end{aligned}
$$

In the Cardy limit, after inserting $\Delta_{R}=2 \pi i+\omega_{1}+\omega_{2}+\omega_{3}$, the index be expanded as follows,

$$
\begin{aligned}
& \log I_{\mathrm{U}(1)}=\sum_{n=1}^{\infty}\left[\frac{(-1)^{n} e^{n \Delta_{L} / 2}+(-1)^{n} e^{-n \Delta_{L} / 2}-2}{n^{4} \omega_{1} \omega_{2} \omega_{3}}\right. \\
& +\frac{-4 \omega_{1}^{2}-4 \omega_{2}^{2}-4 \omega_{2}^{2}+12 \omega_{1} \omega_{2}+12 \omega_{2} \omega_{3}+12 \omega_{3} \omega_{1}-(-1)^{n}\left(e^{n \Delta_{L} / 2}+e^{-n \Delta_{L} / 2}\right)\left(\omega_{1}^{2}+\omega_{2}^{2}+\omega_{3}^{2}\right.}{\left.24 n^{2} \omega_{1} \omega_{2} \omega_{3}\right)} \\
& \left.-\frac{1}{2 n}+\mathcal{O}(\omega)\right] .
\end{aligned}
$$

The $n$ summation becomes divergent after $\mathcal{O}\left(\omega^{-1}\right)$. This divergence signals the breakdown of the perturbative expansion of small $\omega$ after $\mathcal{O}\left(\omega^{-1}\right)$. We expect that the free energy has $\mathcal{O}(\log \omega)$ correction after $\mathcal{O}\left(\omega^{-1}\right)$ order which is consistent with our assumption of the zero-modes. The $\mathcal{O}(\log \omega)$ correction can be explicitly seen in the index if we unrefine the chemical potentials as $\omega_{1,2,3}=\omega$ and $\Delta_{L}=2 \pi i$. Then the modified index (3.34) can be written as follows,

$$
I_{\text {unrefined }}=\exp \sum_{n=1}^{\infty} \frac{1}{n}\left[\frac{2 q^{3 n}-3 q^{4 n}+q^{6 n}}{\left(1-q^{2 n}\right)^{3}}\right]=\prod_{k=3}^{\infty}\left(q^{k} ; q^{2}\right)^{(-1)^{k}(k-1)}, \quad q=e^{-\omega / 2}
$$

where the q-Pochhammer symbol is defined as $(a ; q)=\prod_{k=0}^{\infty}\left(1-a q^{k}\right)$. The asymptotic expansion of the q-Pochhammer symbol for small $\omega$ is [52]

$$
\left(q^{k} ; q^{2}\right)=\exp \left[-\frac{\pi^{2}}{6 \omega}+\frac{1-k}{2} \log \omega+\mathcal{O}\left(\omega^{0}\right)\right] .
$$

Using (3.37), the infinite product over $k$ in (3.36) can be well-regulated, and we obtain the following asymptotic expansion of the unrefined free energy,

$$
\log I_{\text {unrefine }}=\exp \left[\frac{\pi^{2}}{8 \omega}+\frac{1}{2} \log \omega+\mathcal{O}\left(\omega^{0}\right)\right]
$$


which is consistent with the perturbative expansion (3.35) up to $\mathcal{O}\left(\omega^{-1}\right)$. Therefore, we will trust our perturbative expansion (3.35) up to $\mathcal{O}\left(\omega^{-1}\right)$, keeping in mind the existence of $\mathcal{O}(\log \omega)$ correction.

The infinite sum over $n$ in (3.35) can be done with the polylogarithm functions as follows,

$$
\begin{aligned}
\log I_{\mathrm{U}(1)}= & \frac{1}{\omega_{1} \omega_{2} \omega_{3}}\left(\operatorname{Li}_{4}\left(-e^{\Delta_{L} / 2}\right)+\operatorname{Li}_{4}\left(-e^{-\Delta_{L} / 2}\right)-\frac{\pi^{4}}{45}\right) \\
& +\frac{2 \pi^{2}\left(-\omega_{1}^{2}-\omega_{2}^{2}-\omega_{3}^{2}+3 \omega_{1} \omega_{2}+3 \omega_{2} \omega_{3}+3 \omega_{3} \omega_{1}\right)}{72 \omega_{1} \omega_{2} \omega_{3}} \\
& -\frac{3\left(\omega_{1}^{2}+\omega_{2}^{2}+\omega_{3}^{2}\right)\left(\operatorname{Li}_{2}\left(-e^{\Delta_{L} / 2}\right)+\operatorname{Li}_{2}\left(-e^{-\Delta_{L} / 2}\right)\right)}{72 \omega_{1} \omega_{2} \omega_{3}} \\
& +\mathcal{O}(\log \omega) .
\end{aligned}
$$

We can further simplify the above expression by using the following polylogarithm identities,

$$
\operatorname{Li}_{m}\left(-e^{x}\right)+(-1)^{m} \operatorname{Li}_{m}\left(-e^{-x}\right)=-\frac{(2 \pi i)^{m}}{m !} B_{m}\left(\frac{1}{2}+\frac{x}{2 \pi i}\right), \quad-\pi<\operatorname{Im}[x]<\pi
$$

where $B_{m}$ is the $m^{\prime}$ th Bernoulli polynomial defined as $\frac{t e^{x t}}{e^{t}-1}=\sum_{n=0}^{\infty} B_{n}(x) \frac{t^{n}}{n !}$. In the canonical chamber $-2 \pi<\operatorname{Im}\left[\Delta_{L}\right]<2 \pi(3.30)$, the index takes the following form,

$$
\begin{aligned}
\log I_{\mathrm{U}(1)}= & -\frac{1}{384} \frac{\left(\Delta_{L}^{2}+4 \pi^{2}\right)^{2}}{\omega_{1} \omega_{2} \omega_{3}} \\
& +\frac{\left(\omega_{1}^{2}+\omega_{2}^{2}+\omega_{3}^{2}\right) \Delta_{L}^{2}-4 \pi^{2}\left(\omega_{1}^{2}+\omega_{2}^{2}+\omega_{3}^{2}-4 \omega_{1} \omega_{2}-4 \omega_{2} \omega_{3}-4 \omega_{3} \omega_{1}\right)}{192 \omega_{1} \omega_{2} \omega_{3}}+\mathcal{O}(\log \omega) .
\end{aligned}
$$

After inserting $\Delta_{R}=2 \pi i+\omega_{1}+\omega_{2}+\omega_{3}$ to the Cardy formula of the free tensor multiplet (3.32), it is straightforward to check that (3.41) and (3.32) are exactly the same up to $\mathcal{O}\left(\omega^{-1}\right)$.

\subsection{E-string theory of arbitrary rank}

For $(1,0)$ theories, there is no simple classification as ADE classification of $(2,0)$ theories. The general $(1,0)$ theories are engineered from F-theory on an elliptic Calabi-Yau threefold, and most of them admit atomic classification [53]. In this subsection and the next subsection, we will focus on two examples of $(1,0)$ theories that can be engineered from M-theory as well.

A rank $N$ E-string theory is the world volume theory of $N$ M5-branes on a M9brane [54, 55]. The transverse space of M5-branes is $\mathbb{R}^{4} \times \mathbb{R}_{>0}$ where $\mathbb{R}^{4}$ is embedded in the M9-brane. The transverse $\mathbb{R}^{4}$ directions host $\mathrm{SO}(4) \simeq \mathrm{SU}(2)_{R} \times \mathrm{SU}(2)_{L}$ global symmetry where $\mathrm{SU}(2)_{R}$ acts as the $(1,0) \mathrm{R}$-symmetry and $\mathrm{SU}(2)_{L}$ acts as a flavor symmetry. Also, there is $E_{8}$ global symmetry from the M9-brane. The superconformal index takes the following definition,

$$
I=\operatorname{Tr}\left[e^{-\omega_{1} J_{1}-\omega_{2} J_{2}-\omega_{3} J_{3}} \cdot e^{-\Delta_{R} Q_{R}-\Delta_{L} Q_{L}} \cdot \prod_{a=1}^{8} e^{-m_{a} F_{a}}\right]
$$


where the chemical potentials are constrained by $\Delta_{R}-\omega_{1}-\omega_{2}-\omega_{3}=2 \pi i$. Also, $F_{a}$ 's are Cartans of $E_{8}$ with chemical potentials $m_{a}$.

The background gauge fields are $\mathbf{A}_{R}$ for $\mathrm{SU}(2)_{R}, \mathbf{A}_{L}$ for $\mathrm{SU}(2)_{L}$, and $\mathbf{A}_{E}$ for $E_{8}$ which take the following forms,

$$
\mathbf{A}_{L, R}=\frac{\Delta^{L, R}}{\beta} T_{\mathrm{SU}(2)} d \tau, \quad \mathbf{A}_{E}=\sum_{a=1}^{8} \frac{m_{a}}{\beta} T_{E_{8}}^{a} d \tau
$$

where $T_{E_{8}}^{a}$ 's are Cartan generators of $E_{8}$ normalized as $\operatorname{tr}_{\mathbf{a d j}}\left[T_{E_{8}}^{a} T_{E_{8}}^{b}\right]=60 \delta_{a b}$. The anomaly polynomial of the rank $N$ E-string theory is given as [14]

$$
P_{8}=\frac{N^{3}-N}{24} p_{2}(N)+\frac{N}{48}\left[p_{2}(N)-p_{2}(T)+\frac{1}{4}\left(p_{1}(T)-p_{1}(N)\right)^{2}\right]+\frac{N}{2}\left(\frac{N}{2} \chi_{4}(N)+I_{4}\right)^{2}
$$

where

$$
I_{4}=\frac{1}{4}\left(\frac{1}{(2 \pi)^{2}} \operatorname{Tr}\left[\mathbf{F}_{E}^{2}\right]+p_{1}(T)+p_{1}(N)\right), \quad \chi_{4}(N)=c_{2}(L)-c_{2}(R)
$$

and $\mathbf{F}_{E}$ is a field strength two-form of $\mathbf{A}_{E}$. The definitions for Pontryagin classes of the normal bundle and the tangent bundle can be found in (2.35) and (3.24). The thermal anomaly polynomial is given by

$$
\begin{aligned}
P_{8}^{T}= & \frac{N^{3}-N}{24} p_{2}(N)+\frac{N}{48}\left[p_{2}(N)-\left(p_{2}(T)-\frac{\mathbf{F}_{T}^{2}}{4 \pi^{2}} p_{1}(T)\right)+\frac{1}{4}\left(p_{1}(N)-p_{1}(T)+\frac{\mathbf{F}_{T}^{2}}{4 \pi^{2}}\right)^{2}\right] \\
& +\frac{N}{2}\left[\frac{N}{2} \chi_{4}(N)+\frac{1}{4}\left(\operatorname{Tr}\left[\mathbf{F}_{E}^{2}\right]+p_{1}(T)-\frac{\mathbf{F}_{T}^{2}}{4 \pi^{2}}+p_{1}(N)\right)\right]^{2}
\end{aligned}
$$

Then the CS action of background fields on $S^{5}$ is given as follows,

$$
W_{\mathrm{CS}}=2 \pi i \int_{\mathcal{N}^{7}} \frac{d \tau+\mathbf{a}}{d \mathbf{a}}\left[P_{8}^{T}-\hat{P}_{8}^{T}\right]
$$

For the background fields introduced above, the CS action can be written as follows,

$$
\begin{aligned}
& W_{\mathrm{CS}}=\frac{i}{256 \pi^{3} \beta^{3}} \int_{S^{5}} \mathbf{a} d \mathbf{a} d \mathbf{a} \cdot\left[-\frac{N^{3}}{3}\left(\Delta_{R}^{2}-\Delta_{L}^{2}\right)^{2}+\frac{N^{2}}{2}\left(\Delta_{R}^{2}-\Delta_{L}^{2}\right)\left(4 \sum_{a} m_{a}^{2}+8 \pi^{2}-\Delta_{R}^{2}-\Delta_{L}^{2}\right)\right. \\
& -\frac{N}{12}\left(3\left(\Delta_{R}^{4}+\Delta_{R}^{4}\right)+8 \Delta_{R}^{2} \Delta_{L}^{2}-40 \pi^{2}\left(\Delta_{R}^{2}+\Delta_{L}^{2}\right)+24\left(8 \pi^{2}-\Delta_{R}^{2}-\Delta_{L}^{2}\right) \sum_{a} m_{a}^{2}\right. \\
& \left.\left.\quad+48\left(\sum_{a} m_{a}^{2}\right)^{2}+224 \pi^{4}\right)\right] \\
& +\frac{i}{3072 \pi^{3} \beta} \int_{S^{5}} \mathbf{a t r}\left[\hat{\mathbf{R}}^{2}\right] \cdot\left[-6 N^{2}\left(\Delta_{R}^{2}-\Delta_{L}^{2}\right)+N\left(40 \pi^{2}-5\left(\Delta_{R}^{2}+\Delta_{L}^{2}\right)+24 \sum_{a} m_{a}^{2}\right)\right] \\
& +W_{\text {grav-CS. }}
\end{aligned}
$$


Again, pure gravitational CS terms can be ignored in the Cardy limit. After evaluating the integral over $S^{5}$, the Cardy free energy can be obtained as follows,

$$
\begin{aligned}
& \log I=\frac{1}{32 \omega_{1} \omega_{2} \omega_{3}}\left[-\frac{N^{3}}{3}\left(\Delta_{R}^{2}-\Delta_{L}^{2}\right)^{2}+\frac{N^{2}}{2}\left(\Delta_{R}^{2}-\Delta_{L}^{2}\right)\left(4 \sum_{a} m_{a}^{2}+8 \pi^{2}-\Delta_{R}^{2}-\Delta_{L}^{2}\right)\right. \\
& -\frac{N}{12}\left(3\left(\Delta_{R}^{4}+\Delta_{L}^{4}\right)+8 \Delta_{R}^{2} \Delta_{L}^{2}-40 \pi^{2}\left(\Delta_{R}^{2}+\Delta_{L}^{2}\right)+24\left(8 \pi^{2}-\Delta_{R}^{2}-\Delta_{L}^{2}\right) \sum_{a} m_{a}^{2}\right. \\
& \left.\left.\quad+48\left(\sum_{a} m_{a}^{2}\right)^{2}+224 \pi^{4}\right)\right] \\
& +\frac{\omega_{1}^{2}+\omega_{2}^{2}+\omega_{3}^{2}}{192 \omega_{1} \omega_{2} \omega_{3}}\left[-6 N^{2}\left(\Delta_{R}^{2}-\Delta_{L}^{2}\right)+N\left(40 \pi^{2}-5\left(\Delta_{R}^{2}+\Delta_{L}^{2}\right)+24 \sum_{a} m_{a}^{2}\right)\right]+\mathcal{O}(\log \omega)
\end{aligned}
$$

where chemical potentials are constrained by $\Delta_{R}-\omega_{1}-\omega_{2}-\omega_{3}=2 \pi i$.

\subsection{M5 branes on ALE singularities}

In this section, we consider another $(1,0) \mathrm{SCFT}$, which is the worldvolume theory of $N$ M5-branes probing $\mathbb{C}^{2} / \Gamma_{G}$ singularity. It has the following quiver theory description,

$$
G_{0}-G_{1}-\ldots-G_{N-1}-G_{N}
$$

where $G_{1, \ldots, N-1}$ are gauge symmetries and $G_{0, N}$ are flavor symmetries of the same simply laced Lie group $G$. The line - denotes a 'conformal matter' which is a weakly coupled hyper multiplet if $G=A_{N}$, but becomes another nontrivial 6d SCFT with a fractionalized M5-brane when $G$ is $D_{N}$ or $E_{N}$ [56]. We also have vector multiplets for each of the gauge node and a tensor multiplet as a center of the mass degrees of freedom. The $6 \mathrm{~d}$ theory has $\mathrm{SU}(2) \mathrm{R}$-symmetry and $G_{0} \times G_{N}$ flavor symmetry. The modified superconformal index is defined as follows,

$$
I=\operatorname{Tr}\left[e^{-\Delta_{R} Q_{R}} \cdot e^{-\omega_{1} J_{1}-\omega_{2} J_{2}-\omega_{3} J_{3}} \cdot \prod_{i=1}^{r_{G}} e^{-x_{i} M_{i}-y_{i} N_{i}}\right]
$$

where chemical potentials are constrained by $\Delta_{R}-\omega_{1}-\omega_{2}-\omega_{3}=2 \pi i$. Here, $M_{i}$ 's are Cartans of $G_{0}$ and $N_{i}$ 's are Cartans of $G_{N} . x_{i}$ 's and $y_{i}$ 's are their chemical potentials respectively.

The background gauge fields $\mathbf{A}_{R}$ for SU(2) $R$ R-symmetry and $\mathbf{A}_{0, N}$ for $G_{0}$ and $G_{N}$ flavor symmetry are turned on as

$$
\mathbf{A}_{R}=\frac{\Delta_{R}}{\beta} T_{\mathrm{SU}(2)} d \tau, \quad \mathbf{A}_{0}=\frac{x_{i}}{\beta} T_{G}^{i} d \tau, \quad \mathbf{A}_{N}=\frac{y_{i}}{\beta} T_{G}^{i} d \tau
$$

where $T_{G}^{i}$ 's are the Cartan generators of $G$. The anomaly polynomial of this theory is given by $[14]$

$$
\begin{aligned}
P_{8}= & \frac{|\Gamma|^{2} N^{3}}{24} c_{2}(R)^{2}-\frac{N}{48} c_{2}(R)\left(|\Gamma|\left(r_{G}+1\right)-1\right)\left(4 c_{2}(R)+p_{1}(T)\right) \\
& -\frac{N}{8(2 \pi)^{2}}|\Gamma| c_{2}(R)\left(\operatorname{Tr}\left[\mathbf{F}_{0}^{2}\right]+\operatorname{Tr}\left[\mathbf{F}_{N}^{2}\right]\right)+\frac{N}{8}\left(\frac{1}{6} c_{2}(R) p_{1}(T)-\frac{1}{6} p_{2}(T)+\frac{1}{24} p_{1}(T)^{2}\right) \\
& -\frac{1}{2} P_{8}^{\mathrm{vec}}\left(\mathbf{F}_{0}\right)-\frac{1}{2} P_{8}^{\mathrm{vec}}\left(\mathbf{F}_{N}\right)-\frac{1}{2(2 \pi)^{4} N}\left(\frac{1}{4} \operatorname{Tr}\left[\mathbf{F}_{0}^{2}\right]-\frac{1}{4} \operatorname{Tr}\left[\mathbf{F}_{N}^{2}\right]\right)^{2}
\end{aligned}
$$




\begin{tabular}{|c|c|c|c|c|c|}
\hline & $\mathrm{SU}(k)$ & $\mathrm{SO}(2 k)$ & $E_{6}$ & $E_{7}$ & $E_{8}$ \\
\hline$r_{G}$ & $k-1$ & $k$ & 6 & 7 & 8 \\
\hline$h_{G}^{\vee}$ & $k$ & $2 k-2$ & 12 & 18 & 30 \\
\hline$d_{G}$ & $k^{2}-1$ & $k(2 k-1)$ & 78 & 133 & 248 \\
\hline$s_{G}$ & $1 / 2$ & 1 & 3 & 6 & 30 \\
\hline$t_{G}$ & $2 k$ & $2 k-8$ & 0 & 0 & 0 \\
\hline$u_{G}$ & 2 & 4 & 6 & 8 & 12 \\
\hline$|\Gamma|$ & $k$ & $4 k-8$ & 24 & 48 & 120 \\
\hline
\end{tabular}

Table 1. Group-theoretic constants for ADE type. We follow the convention of [14].

where $\mathbf{F}_{0, N}$ is a field strength of the flavor symmetry $G_{0, N}$. The anomaly polynomial of a vector multiplet $P_{8}^{\mathrm{vec}}$ is

$$
\begin{aligned}
P_{8}^{\mathrm{vec}}(\mathbf{F})= & -\frac{1}{24}\left(\frac{h_{G}^{\vee}}{(2 \pi)^{4}} \operatorname{Tr}\left[\mathbf{F}^{4}\right]+\frac{6 h_{G}^{\vee}}{(2 \pi)^{2}} c_{2}(R) \operatorname{Tr}\left[\mathbf{F}^{2}\right]+d_{G} c_{2}(R)^{2}\right) \\
& -\frac{1}{48}\left(\frac{h_{G}^{\vee}}{(2 \pi)^{2}} \operatorname{Tr}\left[\mathbf{F}^{2}\right]+d_{G} c_{2}(R)\right) p_{1}(T)-d_{G} \frac{7 p_{1}(T)^{2}-4 p_{2}(T)}{5760} .
\end{aligned}
$$

The various group theoretic parameters are listed in the table 1 .

Now it is straight forward to compute the CS action of the background fields as follows,

$$
W_{\mathrm{CS}}=2 \pi i \int_{\mathcal{N}^{7}} \frac{d t+\mathbf{a}}{d \mathbf{a}}\left[P_{8}^{T}-\hat{P}_{8}^{T}\right]_{\mathbf{A}^{T}=0} .
$$

For the background fields introduced above, the CS action is given as follows,

$$
\begin{aligned}
& W_{\mathrm{CS}}=-\frac{i}{3072 \pi^{3} \beta^{3}} \int_{S^{5}} \mathbf{a} d \mathbf{a} d \mathbf{a} \cdot\left(N^{3}|\Gamma|^{2} \Delta_{R}^{4}\right. \\
& -N\left[2\left(|\Gamma|\left(r_{G}+1\right)-1\right) \Delta_{R}^{4}+4 \Delta_{R}^{2}\left(2 \pi^{2}\left(|\Gamma|\left(r_{G}+1\right)-2\right)+3|\Gamma|\left(T_{2}(x)+T_{2}(y)\right)\right)-32 \pi^{4}\right] \\
& +d_{G} \Delta_{R}^{4}+\Delta_{R}^{2}\left(8 \pi^{2} d_{G}+12 h_{G}\left(T_{2}(x)+T_{2}(y)\right)\right)+8 h_{G}\left(2 \pi^{2}\left(T_{2}(x)+T_{2}(y)\right)+T_{4}(x)+T_{4}(y)\right) \\
& \left.+\frac{112 \pi^{4}}{15} d_{G}-\frac{12}{N}\left(T_{2}(x)-T_{2}(y)\right)^{2}\right) \\
& -\frac{i}{3072 \pi^{3} \beta} \int_{S^{5}} \mathbf{a t r}\left[\hat{\mathbf{R}}^{2}\right] \cdot\left[N\left(|\Gamma|\left(r_{G}+1\right)-2\right) \Delta_{R}^{2}+8 \pi^{2}\right) \\
& \left.\quad-\left(d_{G} \Delta_{R}^{2}+2 h_{G}\left(T_{2}(x)+T_{2}(y)\right)+\frac{4 \pi^{2}}{3} d_{G}\right)\right] \\
& +W_{\text {grav-CS }}
\end{aligned}
$$

where the function $T_{n}$ is defined as

$$
T_{n}(x)=\operatorname{Tr}\left[\sum_{i=1}^{r_{G}}\left(x_{i} T_{G}^{i}\right)^{n}\right] .
$$


Again, the pure gravitational CS terms are ignored in the Cardy limit. Then, we obtain the following expression for the Cardy free energy,

$$
\begin{aligned}
& \log I=-\frac{1}{384 \omega_{1} \omega_{2} \omega_{3}}\left(N^{3}|\Gamma|^{2} \Delta_{R}^{4}\right. \\
& -N\left[2\left(|\Gamma|\left(r_{G}+1\right)-1\right) \Delta_{R}^{4}+4 \Delta_{R}^{2}\left(2 \pi^{2}\left(|\Gamma|\left(r_{G}+1\right)-2\right)+3|\Gamma|\left(T_{2}(x)+T_{2}(y)\right)\right)-32 \pi^{4}\right] \\
& +d_{G} \Delta_{R}^{4}+\Delta_{R}^{2}\left(8 \pi^{2} d_{G}+12 h_{G}\left(T_{2}(x)+T_{2}(y)\right)\right)+8 h_{G}\left(2 \pi^{2}\left(T_{2}(x)+T_{2}(y)\right)+T_{4}(x)+T_{4}(y)\right) \\
& \left.+\frac{112 \pi^{4}}{15} d_{G}-\frac{12}{N}\left(T_{2}(x)-T_{2}(y)\right)^{2}\right) \\
& -\frac{\omega_{1}^{2}+\omega_{2}^{2}+\omega_{3}^{2}}{192 \omega_{1} \omega_{2} \omega_{3}}\left[N\left(\left(|\Gamma|\left(r_{G}+1\right)-2\right) \Delta_{R}^{2}+8 \pi^{2}\right)-\left(d_{G} \Delta_{R}^{2}+2 h_{G}\left(T_{2}(x)+T_{2}(y)\right)+\frac{4 \pi^{2}}{3} d_{G}\right)\right] \\
& +\mathcal{O}(\log \omega)
\end{aligned}
$$

where the chemical potentials constrained by $\Delta_{R}-\omega_{1}-\omega_{2}-\omega_{3}=2 \pi i$.

Now, in order to check our Cardy formula (3.58), let us consider a single M5-brane probing $\mathbb{C}^{2} / \mathbb{Z}_{k}$ singularity which has the following quiver description,

$$
\mathrm{SU}(k)_{0}-\mathrm{SU}(k)_{1} \text {. }
$$

The above quiver theory is described by a single free $(1,0)$ tensor multiplet and free hyper multiplet charged under the two flavor symmetries $\mathrm{SU}(k)_{0,1}$. The superconformal index is given by [57]

$$
\begin{array}{r}
\mathcal{I}=\operatorname{Tr}\left[(-1)^{F} \cdot e^{\left.-\omega_{1} J_{1}-\omega_{2} J_{2}-\omega_{3} J_{3}\right)} \cdot e^{-\Delta_{R} Q_{R}} \cdot \prod_{i=1}^{k-1} e^{-x_{i} M_{i}-y_{i} N_{i}}\right], \quad\left(\Delta_{R}-\omega_{1}-\omega_{2}-\omega_{3}=0\right) \\
=\exp \sum_{n=1}^{\infty} \frac{1}{n} \frac{1}{\left(1-e^{-n \omega_{1}}\right)\left(1-e^{-n \omega_{2}}\right)\left(1-e^{\left.-n \omega_{3}\right)}\right.}\left(-e^{-n\left(\omega_{1}+\omega_{2}\right)}-e^{-n\left(\omega_{2}+\omega_{3}\right)}-e^{-n\left(\omega_{3}+\omega_{1}\right)}\right. \\
\left.\quad+e^{-n\left(\omega_{1}+\omega_{2}+\omega_{3}\right)}+e^{-n \frac{\Delta_{R}}{2}} \chi(n x) \cdot \bar{\chi}(n y)+e^{-n \frac{\Delta_{R}}{2}} \bar{\chi}(n x) \cdot \chi(n y)\right) \cdot(3.60)
\end{array}
$$

Here $Q_{R}$ is the $\mathrm{SU}(2)$ R-symmetry Cartan. $M_{i}$ 's and $N_{i}$ 's are $\mathrm{SU}(k)_{0}$ and $\mathrm{SU}(k)_{1}$ charges respectively. For $k=1$, the index (3.60) reduces to the index of the free $(2,0)$ tensor multiplet (3.33). $\chi$ is the fundamental character of $\mathrm{SU}(k)$, and $\bar{\chi}$ is the anti-fundamental character. It is convenient to define a new set of basis $X^{i}$, s for the $\mathrm{SU}(k)_{0}$ chemical potentials as follows,

$$
x_{i} T_{\text {fund }}^{i}=\operatorname{diag}\left(X_{1}, X_{2}, \ldots, X_{k}\right)
$$

where $X_{i}$ 's satisfy $\sum_{i=1}^{k} X_{k}=0$. We define $Y_{i}^{\prime}$ 's in a similar way as $X_{i}$ 's. Then, the (anti-)fundamental characters are written as

$$
\chi(n x)=\sum_{i=1}^{k} e^{-n X_{k}}, \quad \bar{\chi}(n x)=\sum_{i=1}^{k} e^{n X_{k}} .
$$


The index (3.60) can be turned into the modified index by shifting $\omega_{3} \rightarrow \omega_{3}+2 \pi i$. Then, the modified index is given as follows,

$$
\begin{array}{r}
I=\operatorname{Tr}\left[e^{-\omega_{1} J_{1}-\omega_{2} J_{2}-\omega_{3} J_{3}} \cdot e^{-\Delta_{R} Q_{R}} \cdot \prod_{i=1}^{k-1} e^{-x_{i} M_{i}-y_{i} N_{i}}\right], \quad\left(\Delta_{R}-\omega_{1}-\omega_{2}-\omega_{3}=2 \pi i\right) \\
=\exp \sum_{n=1}^{\infty} \frac{1}{n} \frac{1}{\left(1-e^{-n \omega_{1}}\right)\left(1-e^{-n \omega_{2}}\right)\left(1-e^{\left.-n \omega_{3}\right)}\right.}\left(e^{-n\left(\omega_{1}+\omega_{2}+\omega_{3}\right)}-e^{-n\left(\omega_{1}+\omega_{2}\right)}-e^{-n\left(\omega_{2}+\omega_{3}\right)}\right. \\
\left.-e^{-n\left(\omega_{3}+\omega_{1}\right)}+e^{-n \frac{\Delta_{R}}{2}} \chi[n x] \cdot \bar{\chi}[n y]+e^{-n \frac{\Delta_{R}}{2}} \bar{\chi}[n x] \cdot \chi[n y]\right) \cdot
\end{array}
$$

The small $\omega$ expansion of the free energy is

$$
\begin{aligned}
\log I=\sum_{n=1}^{\infty}[ & \frac{-2+(-1)^{n}(\chi[n x] \cdot \bar{\chi}[n y]+\bar{\chi}[n x] \cdot \chi[n y])}{n^{4} \omega_{1} \omega_{2} \omega_{3}} \\
& +\frac{1}{24 n^{2} \omega_{1} \omega_{2} \omega_{3}}\left(-4 \omega_{1}^{2}-4 \omega_{2}^{2}-4 \omega_{3}^{2}+12 \omega_{1} \omega_{2}+12 \omega_{2} \omega_{3}+12 \omega_{3}\right. \\
& \left.\left.\quad-(-1)^{n}\left(\omega_{1}^{2}+\omega_{2}^{2}+\omega_{3}^{2}\right)(\chi[n x] \cdot \bar{\chi}[n y]+\bar{\chi}[n x] \cdot \chi[n y])\right)-\frac{1}{2 n}+\mathcal{O}(\omega)\right] .
\end{aligned}
$$

Again, we encounter a divergent term which signals the breakdown of the perturbative expansion after $\mathcal{O}\left(\omega^{-1}\right)$ order. As we have seen, the subleading terms after $\mathcal{O}\left(\omega^{-1}\right)$ contain $\mathcal{O}(\log \omega)$ correction. However, the terms up to $\mathcal{O}\left(\omega^{-1}\right)$ is sufficient to check the validity of (3.58). The infinite $n$ summations can be done by using the polylogarithm functions as follows,

$$
\begin{aligned}
\log I= & \frac{1}{\omega_{1} \omega_{2} \omega_{3}}\left[-\frac{\pi^{4}}{45}+\sum_{i, j=1}^{k}\left(\operatorname{Li}_{4}\left(-e^{-X_{i}+Y_{j}}\right)+\operatorname{Li}_{4}\left(-e^{X_{i}-Y_{j}}\right)\right)\right] \\
+\frac{1}{24 \omega_{1} \omega_{2} \omega_{3}} & {\left[\frac{\pi^{2}}{6}\left(-4 \omega_{1}^{2}-4 \omega_{2}^{2}-4 \omega_{3}^{2}+12 \omega_{1} \omega_{2}+12 \omega_{2} \omega_{3}+12 \omega_{3} \omega_{1}\right)\right.} \\
& \left.-\left(\omega_{1}^{2}+\omega_{2}^{2}+\omega_{3}^{2}\right) \sum_{i, j=1}^{k}\left(\operatorname{Li}_{2}\left(-e^{-X_{i}+Y_{j}}\right)+\operatorname{Li}_{2}\left(-e^{X_{i}-Y_{j}}\right)\right)\right]+\mathcal{O}(\log \omega)
\end{aligned}
$$

It can be further simplified by using the polylogarithm identity (3.40). In the canonical chamber where $-\pi<\operatorname{Im}\left[X_{i}-Y_{j}\right]<\pi$, the Cardy free energy can be written as follows,

$$
\begin{aligned}
\log I= & \frac{1}{\omega_{1} \omega_{2} \omega_{3}}\left[-\frac{\pi^{4}}{45}-\frac{(2 \pi i)^{4}}{4 !} \sum_{i, j=1}^{k} B_{4}\left(\frac{1}{2}+\frac{X_{i}-Y_{j}}{2 \pi i}\right)\right] \\
+\frac{1}{24 \omega_{1} \omega_{2} \omega_{3}}[ & \frac{\pi^{2}}{6}\left(-4 \omega_{1}^{2}-4 \omega_{2}^{2}-4 \omega_{3}^{2}+12 \omega_{1} \omega_{2}+12 \omega_{2} \omega_{3}+12 \omega_{3} \omega_{1}\right) \\
& \left.+\left(\omega_{1}^{2}+\omega_{2}^{2}+\omega_{3}^{2}\right) \cdot \frac{(2 \pi i)^{2}}{2 !} \sum_{i, j=1}^{k} B_{2}\left(\frac{1}{2}+\frac{X_{i}-Y_{j}}{2 \pi i}\right)\right]+\mathcal{O}(\log \omega)
\end{aligned}
$$


Now, let us compare (3.66) with our result (3.58). For $G=\mathrm{SU}(k), T_{n}(x)$ in (3.58) can be written as

$$
\begin{aligned}
& T_{2}(x)=\frac{1}{s_{G}} \operatorname{tr}_{\text {fund }}\left[\left(x_{i} T^{i}\right)^{2}\right]=2 \sum_{i=1}^{k} X_{i}^{2} \\
& T_{4}(x)=\frac{t_{G}}{h_{G}^{\vee}} \operatorname{tr}_{\text {fund }}\left[\left(x_{i} T^{i}\right)^{4}\right]+\frac{3 u_{G}}{4 h_{G}^{\vee} s_{G}^{2}} \operatorname{tr}_{\text {fund }}\left[\left(x_{i} T^{i}\right)^{2}\right]^{2}=2 \sum_{i=1}^{k} X_{i}^{4}+\frac{6}{k}\left(\sum_{i=1}^{k} X_{i}^{2}\right)^{2}
\end{aligned}
$$

where we used the trace identities in [14]. Then we can check that (3.66) and (3.58) are exactly the same up to $\mathcal{O}\left(\omega^{-1}\right)$ order.

\section{Equivariant integral of the thermal anomaly polynomial}

In [16], it was conjectured that the supersymmetric Casimir energy can be obtained from the equivariant integral of the anomaly polynomial. In this section, we apply their conjecture to the Cardy formula such that it is given by the equivariant integral of the thermal anomaly polynomial. As a result, we find that the Cardy formula and the equivariant integral of the thermal anomaly polynomial are related as

$$
\log I=-\int P_{8}^{T}+\mathcal{O}(\log \omega)
$$

where $\int$ denotes the equivariant integral. Note that the Cardy limit $\left|\omega_{i}\right| \ll 1$ is assumed.

The equivariant integral is defined from the equivariant cohomology, where the de Rham differential is twisted with the equivariant parameters. The anomaly polynomial $P_{d+2}$ can be viewed as an equivariant form on $\mathbb{R}^{d}$ with equivariant parameters given by the chemical potentials of the Cartan subalgebra of the global symmetries. See [16] for the detailed explanation. The equivariant integral of $P_{d+2}$ is evaluated by the DuistermaatHeckman formula [58] given as follows,

$$
\int P_{d+2}=\left.\sum_{p \in \text { fixed points }} \frac{P_{d+2}}{e(T M)}\right|_{p}
$$

where $e(T M)$ is the equivariant Euler class. In our case, there is only a single fixed point $p_{0}$, and the 0-form components of the equivariant forms at the fixed point are given as follows [16],

$$
\begin{aligned}
\left.e(T M)\right|_{p_{0}} & =\omega_{1} \omega_{2} \omega_{3}, & \left.c_{2}(R)\right|_{p_{0}} & =-\frac{\Delta_{R}^{2}}{4} \\
\left.p_{1}(T)\right|_{p_{0}} & =\omega_{1}^{2}+\omega_{2}^{2}+\omega_{3}^{2}, & & \left.p_{2}(T)\right|_{p_{0}}=\omega_{1}^{2} \omega_{2}^{2}+\omega_{2}^{2} \omega_{3}^{2}+\omega_{3}^{2} \omega_{1}^{2}
\end{aligned}
$$

For the thermal anomaly polynomial, there is the fictitious gauge field $\mathbf{A}_{T}$. The Chern class of the fictitious gauge field at the fixed point is given as follows,

$$
\left.\frac{1}{4 \pi^{2}} \mathbf{F}_{T}^{2}\right|_{p_{0}}=-(2 \pi i)^{2}
$$


Then, the equivariant integral of the thermal anomaly polynomial (2.33) is given by

$$
\begin{aligned}
-\int P_{8}^{T}= & -\left(\frac{\mathfrak{a}}{384} \Delta_{R}^{4}+\frac{\mathfrak{b} \pi^{2}}{24} \Delta_{R}^{2}+\frac{2 \mathfrak{c} \pi^{4}}{3}\right) \frac{1}{\omega_{1} \omega_{2} \omega_{3}}+\left(\frac{\mathfrak{b}}{96} \Delta_{R}^{2}+\frac{\pi^{2}(2 \mathfrak{c}+\mathfrak{d})}{6}\right) \frac{\omega_{1}^{2}+\omega_{2}^{2}+\omega_{3}^{2}}{\omega_{1} \omega_{2} \omega_{3}} \\
& -\frac{\mathfrak{d}}{24} \frac{\omega_{1}^{2} \omega_{2}^{2}+\omega_{2}^{2} \omega_{3}^{2}+\omega_{3}^{2} \omega_{1}^{2}}{\omega_{1} \omega_{2} \omega_{3}}
\end{aligned}
$$

Comparing (4.5) with the Cardy formula (2.52), we can check that our relation (4.1) is valid. Note that $\mathcal{O}\left(\omega^{1}\right)$ term in the equivariant integral can be ignored when computing the Cardy free energy since it is subleading than $\mathcal{O}(\log \omega)$ correction in (4.1).

Now, let us consider $6 \mathrm{~d}(2,0)$ SCFTs with ADE type. The equivariant integral of the thermal anomaly polynomial of $6 \mathrm{~d}(2,0)$ SCFT $(3.25)$ is given by,

$$
\begin{aligned}
-\int P_{8}^{T}= & -\frac{h_{G}^{\vee} d_{G}+r_{G}}{384} \frac{\left(\Delta_{R}^{2}-\Delta_{L}^{2}\right)^{2}}{\omega_{1} \omega_{2} \omega_{3}}-r_{G}\left[\frac{\left(\Delta_{R}^{2}+4 \pi^{2}\right)\left(\Delta_{L}^{2}+4 \pi^{2}\right)}{192 \omega_{1} \omega_{2} \omega_{3}}\right. \\
& \left.-\frac{\omega_{1}^{2}+\omega_{2}^{2}+\omega_{3}^{2}}{\omega_{1} \omega_{2} \omega_{3}} \frac{\Delta_{R}^{2}+\Delta_{L}^{2}-8 \pi^{2}}{192}+\frac{\omega_{1}^{4}+\omega_{2}^{4}+\omega_{3}^{4}-2 \omega_{1}^{2} \omega_{2}^{2}-2 \omega_{2}^{2} \omega_{3}^{2}-2 \omega_{3}^{2} \omega_{1}^{2}}{192 \omega_{1} \omega_{2} \omega_{3}}\right] .
\end{aligned}
$$

Comparing (4.6) with the Cardy formula (3.29), we can check that our relation (4.1) is valid for general $(2,0)$ theories.

Now, we move on to the $6 \mathrm{~d}(1,0)$ theories. For the rank $N$ E-string theory, the equivariant integral of the thermal anomaly polynomial is given by,

$$
\begin{aligned}
& -\int P_{8}^{T}=\frac{1}{32 \omega_{1} \omega_{2} \omega_{3}}\left[-\frac{N^{3}}{3}\left(\Delta_{R}^{2}-\Delta_{L}^{2}\right)^{2}+\frac{N^{2}}{2}\left(\Delta_{R}^{2}-\Delta_{L}^{2}\right)\left(4 \sum_{a} m_{a}^{2}+8 \pi^{2}-\Delta_{R}^{2}-\Delta_{L}^{2}\right)\right. \\
& -\frac{N}{12}\left(3\left(\Delta_{R}^{4}+\Delta_{L}^{4}\right)+8 \Delta_{R}^{2} \Delta_{L}^{2}-40 \pi^{2}\left(\Delta_{R}^{2}+\Delta_{L}^{2}\right)+24\left(8 \pi^{2}-\Delta_{R}^{2}-\Delta_{L}^{2}\right) \sum_{a} m_{a}^{2}\right. \\
& \left.\left.\quad+48\left(\sum_{a} m_{a}^{2}\right)^{2}+224 \pi^{4}\right)\right] \\
& +\frac{\omega_{1}^{2}+\omega_{2}^{2}+\omega_{3}^{2}}{192 \omega_{1} \omega_{2} \omega_{3}}\left[-6 N^{2}\left(\Delta_{R}^{2}-\Delta_{L}^{2}\right)+N\left(40 \pi^{2}-5\left(\Delta_{R}^{2}+\Delta_{L}^{2}\right)+24 \sum_{a} m_{a}^{2}\right)\right] \\
& -N \frac{7\left(\omega_{1}^{2}+\omega_{2}^{2}+\omega_{3}^{2}\right)+10\left(\omega_{1}^{2} \omega^{2}+\omega_{2}^{2} \omega_{3}^{2}+\omega_{3}^{2} \omega_{1}^{2}\right)}{192 \omega_{1} \omega_{2} \omega_{3}} .
\end{aligned}
$$


For M5-branes on ALE singularities, the equivariant integral of the thermal anomaly polynomial is given by,

$$
\begin{aligned}
& -\int P_{8}^{T}=-\frac{1}{384 \omega_{1} \omega_{2} \omega_{3}}\left(N^{3}|\Gamma|^{2} \Delta_{R}^{4}\right. \\
& -N\left[2\left(|\Gamma|\left(r_{G}+1\right)-1\right) \Delta_{R}^{4}+4 \Delta_{R}^{2}\left(2 \pi^{2}\left(|\Gamma|\left(r_{G}+1\right)-2\right)+3|\Gamma|\left(T_{2}(x)+T_{2}(y)\right)\right)-32 \pi^{4}\right] \\
& +d_{G} \Delta_{R}^{4}+\Delta_{R}^{2}\left(8 \pi^{2} d_{G}+12 h_{G}\left(T_{2}(x)+T_{2}(y)\right)\right)+8 h_{G}\left(2 \pi^{2}\left(T_{2}(x)+T_{2}(y)\right)+T_{4}(x)+T_{4}(y)\right) \\
& \left.+\frac{112 \pi^{4}}{15} d_{G}-\frac{12}{N}\left(T_{2}(x)-T_{2}(y)\right)^{2}\right) \\
& -\frac{\omega_{1}^{2}+\omega_{2}^{2}+\omega_{3}^{2}}{192 \omega_{1} \omega_{2} \omega_{3}}\left[N\left(\left(|\Gamma|\left(r_{G}+1\right)-2\right) \Delta_{R}^{2}+8 \pi^{2}\right)-\left(d_{G} \Delta_{R}^{2}+2 h_{G}\left(T_{2}(x)+T_{2}(y)\right)+\frac{4 \pi^{2}}{3} d_{G}\right)\right] \\
& +\frac{N}{192} \frac{2 \omega_{1}^{2} \omega_{2}^{2}+2 \omega_{2}^{2} \omega_{3}^{2}+2 \omega_{3}^{2} \omega_{1}^{2}-\omega_{1}^{4}-\omega_{2}^{4}-\omega_{3}^{4}}{\omega_{1} \omega_{2} \omega_{3}} \\
& -\frac{d_{G}}{5760} \frac{10\left(\omega_{1}^{2} \omega_{2}^{2}+\omega_{2}^{2} \omega_{3}^{2}+\omega_{3}^{2} \omega_{1}^{2}\right)+7\left(\omega_{1}^{4}+\omega_{2}^{4}+\omega_{3}^{4}\right)}{\omega_{1} \omega_{2} \omega_{3}}
\end{aligned}
$$

For all the above results of $6 \mathrm{~d}(1,0)$ theories, we can see that our relation $(4.1)$ is valid by comparing with the Cardy formula (3.49) and (3.58).

Before ending this section, let us make a few comments on the relation between the Cardy formula and the supersymmetric Casimir energy. Under the presence of the Casimir energy, the index function $Z$ can be divided into the Casimir energy part $E_{0}$ and the spectral/state-counting part $I$ as follows.

$$
Z=e^{-E_{0}} I
$$

Casimir energy becomes the dominant contribution to $\log Z$ when the chemical potentials are large. However, our Cardy formula determines the behavior of the spectral part $\log I$ in the Cardy limit where the real parts of the chemical potentials are small. In 6d SCFTs, the Cardy formula and the Casimir energy have similar, but different expressions. Let us explain the difference between two quantities in the following paragraph.

As an example, let us consider the $6 \mathrm{~d}(2,0)$ theory. Its supersymmetric Casimir energy is given by $[16]^{2}$

$$
\begin{aligned}
& E_{0}=-\frac{h_{G}^{\vee} d_{G}+r_{G}}{384} \frac{\left(\Delta_{R}^{2}-\Delta_{L}^{2}\right)^{2}}{\omega_{1} \omega_{2} \omega_{3}} \\
& -r_{G}\left[\frac{\Delta_{R}^{2} \Delta_{L}^{2}}{192 \omega_{1} \omega_{2} \omega_{3}}-\frac{\Delta_{R}^{2}+\Delta_{L}^{2}}{192} \frac{\omega_{1}^{2}+\omega_{2}^{2}+\omega_{3}^{2}}{\omega_{1} \omega_{2} \omega_{3}}+\frac{\omega_{1}^{4}+\omega_{2}^{4}+\omega_{3}^{4}-2 \omega_{1}^{2} \omega_{2}^{2}-2 \omega_{2}^{2} \omega_{3}^{2}-2 \omega_{3}^{2} \omega_{1}^{2}}{192 \omega_{1} \omega_{2} \omega_{3}}\right] .
\end{aligned}
$$

In (4.10), the chemical potentials are defined in the conventional basis as (3.1), which are constrained by $\Delta_{R}-\omega_{1}-\omega_{2}-\omega_{3}=0$. On the other side, our Cardy formula is given

\footnotetext{
${ }^{2}$ The notations can be matched by $\left(2 \beta \sigma_{1}\right)_{\text {there }}=\left(\Delta_{R}+\Delta_{L}\right)_{\text {here }},\left(2 \beta \sigma_{2}\right)_{\text {there }}=\left(\Delta_{R}-\Delta_{L}\right)_{\text {here }}$, and $\left(\beta \omega_{I}\right)_{\text {there }}=\left(\omega_{I}\right)_{\text {here }}$
} 
by (3.29) which is

$$
\begin{aligned}
\log I= & -\frac{h_{G}^{\vee} d_{G}+r_{G}}{384} \frac{\left(\Delta_{R}^{2}-\Delta_{L}^{2}\right)^{2}}{\omega_{1} \omega_{2} \omega_{3}} \\
& -r_{G}\left[\frac{\left(\Delta_{R}^{2}+4 \pi^{2}\right)\left(\Delta_{L}^{2}+4 \pi^{2}\right)}{192 \omega_{1} \omega_{2} \omega_{3}}-\frac{\Delta_{R}^{2}+\Delta_{L}^{2}-8 \pi^{2}}{192} \frac{\omega_{1}^{2}+\omega_{2}^{2}+\omega_{3}^{2}}{\omega_{1} \omega_{2} \omega_{3}}\right]+\mathcal{O}(\log \omega) .
\end{aligned}
$$

In (4.11), the chemical potentials are defined in the modified basis as (3.6), which are constrained by $\Delta_{R}-\omega_{1}-\omega_{2}-\omega_{3}=2 \pi i$.

Although the Casimir energy and the Cardy free energy are defined in the different basis, they share similar expressions. The similarity between the Casimir energy and the Cardy formula was also observed in [25, 31, 38]. However, in $6 \mathrm{~d}$, the subleading corrections in the Cardy formula differ from the Casimir energy. More precisely, the homogeneous degree-one terms of the chemical potentials in the Cardy formula reproduce the Casimir energy up to $\mathcal{O}\left(\omega^{-1}\right)$. Also, the homogenous degree-one terms in the equivariant integral of the thermal anomaly polynomial (4.6) reproduce the entire Casimir energy. In terms of the CS actions classified in (2.17), the homogeneous terms come from $W_{\text {bulk }}$ and $W_{\text {NCS }}$ only. Those CS terms can be entirely determined from the ordinary anomaly polynomial $P_{8}$, while the invariant CS term $W_{\text {ICS }}$ needs the information of the thermal anomaly polynomial $P_{8}^{T}$ to be determined. As a result, the Casimir energy coincides with the homogeneous terms in the Cardy formula up to $\mathcal{O}\left(\omega^{-1}\right)$.

\section{$5 \quad$ Asymptotic entropy}

In this section, we consider the microcanonical ensemble of the 6d SCFTs and compute the asymptotic entropy in the Cardy limit. For $6 \mathrm{~d}(2,0)$ SCFTs of $A_{N}$ and $D_{N}$ type, their large $N$ free energies precisely account the entropy of BPS black holes in the dual $A d S_{7}$. We further consider a general $6 \mathrm{~d}(1,0)$ SCFTs and find a bound on the 't Hooft anomaly coefficients from the non-negativity condition of the entropy.

\subsection{Holographic SCFTs and $A d S_{7}$ black holes}

The large $N$ limit of $6 \mathrm{~d}(2,0)$ SCFTs are known to be dual to eleven-dimensional supergravity on $A d S_{7} \times M^{4}$ where $M^{4}$ should be $S^{4}$ or its orbifolds [59]. The internal manifold $M^{4}$ is given by $S^{4}$ for $A_{N}$ type SCFT and $S^{4} / \mathbb{Z}_{2}$ for $D_{N}$ type SCFT. Here, we compute the degeneracies of BPS states in those holographic SCFTs and compare with the BekensteinHawking entropy of BPS black holes in $A d S_{7}$. The degeneracy $\Omega$ of the BPS states in $6 \mathrm{~d}$ $(2,0)$ SCFTs can be obtained by the inverse Laplace transformation of the index $I$. In the Cardy limit, it can be evaluated by the saddle point approximation which is given by the extremization of the following entropy function,

$$
S=\log I+\Delta_{R} Q_{R}+\Delta_{L} Q_{L}+\omega_{1} J_{1}+\omega_{2} J_{2}+\omega_{3} J_{3}
$$

where $S=\log \Omega$ is the entropy of the BPS states. The extremization should be done over the chemical potentials constrained by $\Delta_{R}-\omega_{1}-\omega_{2}-\omega_{3}=2 \pi i$. 
Let us first discuss the large $N$ limit of the $6 \mathrm{~d}(2,0) A_{N}$ SCFT. In the Cardy limit, its free energy is given by,

$$
\begin{aligned}
\log I_{A_{N}}= & -\frac{(N+1)^{3}-1}{384} \frac{\left(\Delta_{R}^{2}-\Delta_{L}^{2}\right)^{2}}{\omega_{1} \omega_{2} \omega_{3}} \\
& -N\left[\frac{\left(\Delta_{R}^{2}+4 \pi^{2}\right)\left(\Delta_{L}^{2}+4 \pi^{2}\right)}{192 \omega_{1} \omega_{2} \omega_{3}}-\frac{\Delta_{R}^{2}+\Delta_{L}^{2}-8 \pi^{2}}{192} \frac{\omega_{1}^{2}+\omega_{2}^{2}+\omega_{3}^{2}}{\omega_{1} \omega_{2} \omega_{3}}\right]+\mathcal{O}(\log \omega) .
\end{aligned}
$$

In the remaining part of this section, we will assume that $\mathcal{O}(\log \omega)$ correction is subleading than $N^{3}$ in the large $N$. Then, the large $N$ free energy is

$$
\log I_{A_{N}}=-\frac{N^{3}}{384} \frac{\left(\Delta_{R}^{2}-\Delta_{L}^{2}\right)^{2}}{\omega_{1} \omega_{2} \omega_{3}} .
$$

In order to reproduce the black hole entropy from the above large $N$ free energy, we should convert the field-theoretic quantity $N$ to the gravitational quantity $G_{7}$ which is the Newton constant of the dual $A d S_{7}$. It can be computed from the dimensional reduction of the eleven-dimensional supergravity whose Netwon constant is given by $G_{11}=16 \pi^{7} \ell_{P}^{9}$ where $\ell_{P}$ is the Plank length. Since the $11 \mathrm{~d}$ manifold is given by a direct product of $A d S_{7}$ and $S^{4}, G_{7}$ can be obtained by

$$
G_{7}=\frac{G_{11}}{\operatorname{vol}\left(S^{4}\right)}
$$

Let us denote $\ell$ as a radius of $A d S_{7}$. Then, the radius of $S^{4}$ is given by $\ell / 2$. From the quantization of the 4 -form flux on $S^{4}, \ell$ is related to $N$ by [60]

$$
N=\frac{1}{8 \pi} \frac{\ell^{3}}{\ell_{P}^{3}}
$$

Therefore, the $A d S_{7}$ Newton constant is given as follows,

$$
G_{7}=16 \pi^{7} \ell_{P}^{9} \times\left(\frac{8}{3} \pi^{2}\left(\frac{\ell}{2}\right)^{4}\right)^{-1}=\frac{3 \pi^{2} \ell^{5}}{16 N^{3}} .
$$

In terms of $G_{7}$, the free energy of $A_{N}$ SCFT in the large $N$ limit can be written as follows,

$$
\log I_{A_{N}}=-\frac{\pi^{2} \ell^{5}}{2048 G_{7}} \frac{\left(\Delta_{R}^{2}-\Delta_{L}^{2}\right)^{2}}{\omega_{1} \omega_{2} \omega_{3}}
$$

The entropy can be obtained by extremizing the following entropy function,

$$
S=-\frac{\pi^{2} \ell^{5}}{2048 G_{7}} \frac{\left(\Delta_{R}^{2}-\Delta_{L}^{2}\right)^{2}}{\omega_{1} \omega_{2} \omega_{3}}+\Delta_{R} Q_{R}+\Delta_{L} Q_{L}+\omega_{1} J_{1}+\omega_{2} J_{2}+\omega_{3} J_{3} .
$$

The above extremization was first performed in [19], and analytic expressions were derived in [26]. The entropy $S$ in (5.8) can be compared to the entropy of BPS black holes in asymptotic $A d S_{7}$ whose solution was obtained in [61]. The known BPS black hole solution satisfies a certain 'charge relation' which comes from the fact that the BPS black holes exist on the intersection of BPS bound and extremal bound. After imposing the charge 
relation of the BPS black holes, we obtain the following expression for the entropy by extremizing (5.8),

$$
\begin{aligned}
S= & 2 \pi \sqrt{\frac{3\left(Q_{1}^{2} Q_{2}+Q_{1} Q_{2}^{2}\right)-\frac{3 \pi^{2} \ell^{5}}{16 G_{7}}\left(J_{1} J_{2}+J_{2} J_{3}+J_{3} J_{1}\right)}{3\left(Q_{1}+Q_{2}\right)-\frac{3 \pi^{2} \ell^{5}}{16 G_{7}}}} \\
= & 2 \pi\left(\frac{\pi^{2} \ell^{5}}{16 G_{7}}\left(J_{1}+J_{2}+J_{3}\right)+\frac{Q_{1}^{2}+Q_{2}^{2}}{2}+2 Q_{1} Q_{2}\right)^{\frac{1}{2}} \\
& \times\left(1-\sqrt{\left.1-\frac{\frac{\pi^{2} \ell^{5}}{8 G_{7}} J_{1} J_{2} J_{3}+Q_{1}^{2} Q_{2}^{2}}{\left(\frac{\pi^{2} \ell^{5}}{16 G_{7}}\left(J_{1}+J_{2}+J_{3}\right)+\frac{Q_{1}^{2}+Q_{2}^{2}}{2}+2 Q_{1} Q_{2}\right)^{2}}\right)^{\frac{1}{2}}}\right.
\end{aligned}
$$

where $Q_{1} \equiv Q_{R}+Q_{L}$ and $Q_{2} \equiv Q_{R}-Q_{L}$. The equivalence of two expressions in (5.9) is the charge relation of BPS black holes. The obtained entropy (5.9) is exactly the same with the entropy of an $A d S_{7}$ black hole solution obtained in [61].

Now, we consider the large $N$ limit of $D_{N}$ SCFT and its gravity dual. In the Cardy limit, the free energy of the $D_{N}$ theory is

$$
\begin{aligned}
\log I_{D_{N}}= & -\frac{(2 N-2)\left(2 N^{2}-N\right)+N}{384} \frac{\left(\Delta_{R}^{2}-\Delta_{L}^{2}\right)^{2}}{\omega_{1} \omega_{2} \omega_{3}} \\
& -N\left[\frac{\left(\Delta_{R}^{2}+4 \pi^{2}\right)\left(\Delta_{L}^{2}+4 \pi^{2}\right)}{192 \omega_{1} \omega_{2} \omega_{3}}-\frac{\Delta_{R}^{2}+\Delta_{L}^{2}-8 \pi^{2}}{192} \frac{\omega_{1}^{2}+\omega_{2}^{2}+\omega_{3}^{2}}{\omega_{1} \omega_{2} \omega_{3}}\right]+\mathcal{O}(\log \omega) .
\end{aligned}
$$

The large $N$ free energy is

$$
\log I_{D_{N}}=-\frac{N^{3}}{96} \frac{\left(\Delta_{R}^{2}-\Delta_{L}^{2}\right)^{2}}{\omega_{1} \omega_{2} \omega_{3}} .
$$

The corresponding gravity dual theory is eleven-dimensional supergravity on $A d S_{7} \times S^{4} / \mathbb{Z}_{2}$. Due to $\mathbb{Z}_{2}$ orbifold, $\operatorname{vol}\left(S^{4} / \mathbb{Z}_{2}\right)=\operatorname{vol}\left(S^{4}\right) / 2$. Also, the flux quantization on $S^{4} / \mathbb{Z}_{2}$ is changed as

$$
N=\frac{1}{16 \pi} \frac{\ell^{3}}{\ell_{P}^{3}} .
$$

And the Newton constant on $A d S_{7}$ is given by

$$
G_{7}=\frac{G_{11}}{\operatorname{vol}\left(S^{4} / \mathbb{Z}_{2}\right)}=\frac{3 \pi^{2} \ell^{5}}{64 N^{3}} .
$$

Therefore the large $N$ free energy of $D_{N}$ theory can be written as

$$
\log I_{D_{N}}=-\frac{\pi^{2} \ell^{5}}{2048 G_{7}} \frac{\left(\Delta_{R}^{2}-\Delta_{L}^{2}\right)^{2}}{\omega_{1} \omega_{2} \omega_{3}}
$$

In $A d S_{7}$ gravity, (5.14) has the same form with (5.7), thus accounting the same macroscopic entropy of the $A d S_{7}$ black hole.

Before concluding this section, let us make some comments on the large $N$ free energies and the Cardy limit. The obtained large $N$ free energies (5.3) and (5.11) are seemingly the leading order $\mathcal{O}\left(\omega^{-3}\right)$ in the Cardy limit, but actually they include the subleading terms up to $\mathcal{O}\left(\omega^{1}\right)$ since $\Delta_{R}=2 \pi i+\omega_{1}+\omega_{2}+\omega_{3}$. Even considering $\mathcal{O}(\log \omega)$ correction, (5.3) and (5.11) are exact up $\mathcal{O}\left(\omega^{-1}\right)$. Therefore, our Cardy series expansion yields the correct entropy function of $A d S_{7}$ black holes beyond the leading order. 


\subsection{Bound on the anomaly coefficients}

In this section, we consider a general $6 \mathrm{~d}(1,0)$ SCFT without flavor symmetry and analyze its entropy in the Cardy limit. Let us consider the following form of the modified superconformal index,

$$
I=\operatorname{Tr}\left[e^{-\Delta_{R} Q_{R}} \cdot e^{-\omega_{1} J_{1}-\omega_{2} J_{2}-\omega_{3} J_{3}}\right], \quad \Delta_{R}-\sum_{I=1}^{3} \omega_{I}=2 \pi i .
$$

where all notations are the same with section 3 . The anomaly polynomial of the $6 \mathrm{~d}$ SCFT with $\mathrm{SU}(2)_{R}$ R-symmetry and the tangent bundle can be written as follows,

$$
P_{8}=\frac{1}{4 !}\left(\mathfrak{a} \cdot c_{2}(R)^{2}+\mathfrak{b} \cdot c_{2}(R) p_{1}(T)+\mathfrak{c} \cdot p_{1}(T)^{2}+\mathfrak{d} \cdot p_{2}(T)\right)
$$

where $\mathfrak{a}, \mathfrak{b}, \mathfrak{c}$ and $\mathfrak{d}$ are the anomaly coefficients of the theory. The Chern class and the Pontryagin classes are defined in (2.34) and (2.35). From the anomaly polynomial (5.16), we obtain the Cardy free energy (2.52) as follows,

$$
\log I=-\left(\frac{\mathfrak{a}}{384} \Delta_{R}^{4}+\frac{\mathfrak{b} \pi^{2}}{24} \Delta_{R}^{2}+\frac{2 \mathfrak{c} \pi^{4}}{3}\right) \frac{1}{\omega_{1} \omega_{2} \omega_{3}}+\left(\frac{\mathfrak{b}}{96} \Delta_{R}^{2}+\frac{(2 \mathfrak{c}+\mathfrak{d}) \pi^{2}}{6}\right) \frac{\omega_{1}^{2}+\omega_{2}^{2}+\omega_{3}^{2}}{\omega_{1} \omega_{2} \omega_{3}}+\mathcal{O}(\log \omega) .
$$

which was derived in section 2. The asymptotic entropy of the SCFT can be obtained by extremizing the following entropy function with respect to the chemical potentials,

$$
S=\log I+\Delta_{R} Q_{R}+\sum_{I=1}^{3} \omega_{I} J_{I}
$$

where chemical potentials are constrained by $\Delta_{R}-\omega_{1}-\omega_{2}-\omega_{3}=2 \pi i$. Once we impose this constraint, the entropy function becomes,

$$
S=-\frac{\pi^{4}(\mathfrak{a}-4 \mathfrak{b}+16 \mathfrak{c})}{24 \omega_{1} \omega_{2} \omega_{3}}+\mathcal{O}\left(\omega^{-2}\right)+\sum_{I=1}^{3} \omega_{I}\left(J_{I}+Q\right)+2 \pi i Q
$$

where we keep only the leading term in ther Cardy limit. Now, let us consider the equal chemical potential setting $\omega \equiv \omega_{1,2,3}$ for the simplicity. Then, the entropy function is

$$
S=-\frac{\pi^{4}(\mathfrak{a}-4 \mathfrak{b}+16 \mathfrak{c})}{24 \omega^{3}}+\mathcal{O}\left(\omega^{-2}\right)+3 \omega(J+Q)+2 \pi i Q .
$$

where $J \equiv J_{1,2,3}$. The extremization equation $\frac{\partial S}{\partial \omega}=0$ determines the charge as the function of the chemical potentials as follows,

$$
J+Q=-\frac{\pi^{4}(\mathfrak{a}-4 \mathfrak{b}+16 \mathfrak{c})}{24 \omega^{4}}+\mathcal{O}\left(\omega^{-3}\right) .
$$

Let us denote the real and the imaginary part of the chemical potential explicitly as $\omega=$ $\omega_{R}+i \omega_{I}$ where $\omega_{I} \in \mathbb{R}$ and $\omega_{R}>0$. Then, we can separate the real and the imaginary part of (5.21) as follows,

$$
J+Q=-\frac{\pi^{4}(\mathfrak{a}-4 \mathfrak{b}+16 \mathfrak{c})}{24|\omega|^{8}}\left(\left(\omega_{R}^{4}-6 \omega_{R}^{2} \omega_{I}^{2}+\omega_{I}^{6}\right)+4 i \omega_{R} \omega_{I}\left(\omega_{I}^{2}-\omega_{R}^{2}\right)\right)+\mathcal{O}\left(\omega^{-3}\right) .
$$




\begin{tabular}{|c|c|c|c|c|c|}
\hline & $\mathfrak{a}$ & $\mathfrak{b}$ & $\mathfrak{c}$ & $\mathfrak{d}$ & $\mathfrak{a}-4 \mathfrak{b}+16 \mathfrak{c}$ \\
\hline$(1,0)$ tensor & 1 & $\frac{1}{2}$ & $\frac{23}{240}$ & $-\frac{29}{60}$ & $\frac{8}{15}$ \\
\hline$(1,0)$ hyper & 0 & 0 & $\frac{7}{240}$ & $-\frac{1}{60}$ & $\frac{7}{15}$ \\
\hline$(2,0)$ tensor & 1 & $\frac{1}{2}$ & $\frac{1}{8}$ & $-\frac{1}{2}$ & 1 \\
\hline$(2,0) \mathrm{ADE}$ & $h_{G}^{\vee} d_{G}+r_{G}$ & $\frac{r_{G}}{2}$ & $\frac{r_{G}}{8}$ & $-\frac{r_{G}}{2}$ & $h_{G}^{\vee} d_{G}+r_{G}$ \\
\hline rank $N$ E-string & $4 N^{3}-6 N^{2}+3 N$ & $3 N^{2}-\frac{5}{2} N$ & $\frac{7}{8} N$ & $-\frac{N}{2}$ & $4 N^{3}-18 N^{2}+27 N$ \\
\hline$N$ M5's on $\mathbb{C}^{2} / \Gamma_{G}$ & $\begin{array}{c}|\Gamma|^{2} N^{3} \\
-2 N\left(\left(r_{G}+1\right)|\Gamma|-1\right)+d_{G}\end{array}$ & $N\left(1-\frac{r_{G}+1}{2}|\Gamma|\right)+\frac{d_{G}}{2}$ & $\frac{N}{8}+\frac{7 d_{G}}{240}$ & $-\frac{N}{2}-\frac{d_{G}}{60}$ & $|\Gamma|^{2} N^{3}-\frac{8 d_{G}}{15}$ \\
\hline
\end{tabular}

Table 2. Anomaly coefficients of free multiplets and various SCFTs in 6d.

Since the charge should be real, we obtain $\omega_{I}$ as a function of $\omega_{R}$ as follows,

$$
\omega_{I}= \pm \omega_{R}+\mathcal{O}\left(\omega_{R}^{2}\right)
$$

If we plug (5.23) and (5.22) to (5.20), we obtain the real part of the entropy as follows,

$$
\operatorname{Re}[S]=\frac{\pi^{4}(\mathfrak{a}-4 \mathfrak{b}+16 \mathfrak{c})}{24 \omega_{R}^{3}}+\mathcal{O}\left(\omega_{R}^{-2}\right)
$$

As a result, the real part of the entropy (5.24) is non-negative as long as the anomaly coefficients satisfy the following bound,

$$
\mathfrak{a}-4 \mathfrak{b}+16 \mathfrak{c} \geq 0
$$

In table 2, we tabulated the anomaly coefficients of $6 \mathrm{~d}$ free multiplets and various SCFTs. As can be seen, the entropy coefficient $\mathfrak{a}-4 \mathfrak{b}+16 \mathfrak{c}$ is positive for all examples analyzed in this paper.

\section{Concluding remarks}

This paper considered the modified superconformal indices of 6d SCFTs in the Cardy limit and computed the $6 \mathrm{~d}$ Cardy formulas from the $6 \mathrm{~d}$ anomaly polynomials. We tested our results with the $6 \mathrm{~d}$ free SCFTs whose closed forms of the superconformal indices are known. For the $(2,0) A_{N^{-}}$and $D_{N}$-type theories, our Cardy formulas scale as $\mathcal{O}\left(N^{3}\right)$ in the large $N$ limit, and they reproduce the Bekenstein-Hawking entropy of the black holes in dual $A d S_{7}$. We further conjecture the inequality of the $6 \mathrm{~d}$ anomaly coefficients that all $6 \mathrm{~d}$ SCFTs should follow.

Let us conclude this paper with a few remarks. In this paper, our $6 \mathrm{~d}$ superconformal Cardy formulas could be tested with the superconformal indices of $6 \mathrm{~d}$ free SCFTs only. For the interacting SCFTs, the $6 \mathrm{~d}$ Cardy formulas were computed for the $\mathbb{R}^{4} \times T^{2}$ indices in $[62,63]$ using the elliptic genus method, and the authors checked that the results are the same with the background field method on $\mathbb{R}^{4} \times T^{2}$. We hope that we can further check our Cardy formulas with the superconformal indices of 6 d interacting SCFTs on $S^{5} \times S^{1}$.

Lastly, let us comment on the possible modification of the Cardy formula. In [34], the authors computed the $4 \mathrm{~d}$ Cardy formula at real chemical potentials, and it takes the 
form of $\log I \propto c-a$ where $a$ and $c$ are $4 \mathrm{~d}$ central charges. However, in [64], it was pointed out that the Cardy formula can receive corrections for the theories with $a>c$. Such corrections seem non-universal, which means they cannot be universally written in terms of the central charges. Currently, there is no reason to say that such modification does not happen in $6 \mathrm{~d}$. However, our $6 \mathrm{~d}$ Cardy formula only depends on the anomaly coefficients, which are related to the $6 \mathrm{~d}$ central charges [65]. Therefore, it seems that such non-universal modification of the Cardy formula cannot be captured by the background field method. It would be important to clarify the regime where our Cardy formula is valid if such modification happens in $6 \mathrm{~d}$.

\section{Acknowledgments}

We thank Nikolay Bobev, Sunjin Choi, Joonho Kim, Jung-Wook Kim, Ki-Hong Lee, Jaewon Song, and Yang Zhou for the helpful discussion and comments. We especially appreciate Seok Kim for suggesting the project, giving inspiring comments, and carefully reading the manuscript. This work is supported by the National Research Foundation of Korea (NRF) Grant 2018R1A2B6004914, the Hyundai Motor Chung Mong-Koo Foundation.

\section{A A $4 \mathrm{~d} \mathcal{N}=1$ Cardy formula}

In this section, we reproduce a $4 \mathrm{~d} \mathcal{N}=1$ Cardy formula found in [37, 38] by following our anomaly-based approach. We consider the following superconformal index of a $4 \mathrm{~d} \mathcal{N}=1$ theory,

$$
I=\operatorname{Tr}\left[e^{-\Delta R} \cdot e^{-\omega_{1} J_{1}-\omega_{2} J_{2}}\right], \quad\left(2 \Delta-\omega_{1}-\omega_{2}=2 \pi i\right)
$$

where $R$ is a charge of $\mathrm{U}(1)$ R-symmetry and $J_{1,2}$ are angular momenta on $S^{3}$. The background metric of $S^{3} \times S^{1}$ is given by [26]

$$
\begin{aligned}
d s^{2} & =d \tau^{2}+r^{2}\left[d \theta^{2}+\sum_{i=1}^{2} n_{i}^{2} \theta\left(d \phi_{i}-\frac{i \omega_{i}}{\beta} d \tau\right)^{2}\right], \quad\left(n_{1}, n_{2}\right)=(\cos \theta, \sin \theta) \\
& =e^{-2 \Phi}(d \tau+\mathbf{a})^{2}+r^{2}\left[d \theta^{2}+\sum_{i=1}^{2} n_{i}^{2} d \phi_{i}^{2}+\frac{r^{2} \sum_{i} \omega_{i} n_{i}^{2} d \phi_{i}}{\beta^{2}\left(1-r^{2} \sum_{i} \frac{n_{i}^{2} \omega_{i}^{2}}{\beta^{2}}\right)}\right]
\end{aligned}
$$

where $r$ is a radius of $S^{3}$ and $\tau \sim \tau+\beta$. The dilaton $\Phi$ and the graviphoton a are

$$
e^{-2 \Phi}=1-r^{2} \sum_{i=1}^{2} \frac{n_{i}^{2} \omega_{i}^{2}}{\beta^{2}}, \quad \mathbf{a}=-i \frac{r^{2} \sum_{i} \omega_{i} n_{i}^{2} d \phi_{i}}{\beta\left(1-r^{2} \sum_{i} \frac{n_{i}^{2} \omega_{i}^{2}}{\beta^{2}}\right)}
$$

The background U(1) R-symmetry field is given by

$$
\mathbf{A}=\frac{\Delta}{\beta} d \tau
$$

The anomaly polynomial of $4 \mathrm{~d} \mathcal{N}=1$ theories has the following form,

$$
P_{6}=\frac{1}{6} k_{R R R} c_{1}(R)^{3}-\frac{1}{24} k_{R} c_{1}(R) p_{1}(T), \quad c_{1}(R)=\frac{i}{2 \pi} d \mathbf{A}
$$


where $c_{1}(R)$ is the first Chern-class of $\mathrm{U}(1) \mathrm{R}$-symmetry and $p_{1}(T)$ is the first Pontryagin class of a tangent bundle. Two central charges $a$ and $c$ of $4 \mathrm{~d}$ SCFTs are related to the 't Hooft anomaly coefficients as follows [66],

$$
a=\frac{3}{32}\left(3 k_{R R R}-k_{R}\right), \quad c=\frac{1}{32}\left(9 k_{R R R}-5 k_{R}\right)
$$

Then the anomaly polynomial can be written in terms of the central charges as follows.

$$
P_{6}=\frac{8(5 a-3 c)}{27} c_{1}(R)^{3}-\frac{2(a-c)}{3} c_{1}(R) p_{1}(T)
$$

The thermal anomaly polynomial $P_{6}^{T}$ can be obtained from the replacement rule (2.19) as follows,

$$
P_{6}^{T}=\frac{8(5 a-3 c)}{27} c_{1}(R)^{3}-\frac{2(a-c)}{3} c_{1}(R)\left(p_{1}(T)-\frac{\mathbf{F}_{T}^{2}}{4 \pi^{2}}\right)
$$

The CS action on $S^{3}$ is

$$
W_{\mathrm{CS}}=2 \pi i \int_{\mathcal{N}^{5}} \frac{d t+\mathbf{a}}{d \mathbf{a}}\left[P_{6}^{T}-\hat{P}_{6}^{T}\right]_{\mathbf{A}^{T}=0}
$$

$\mathcal{N}^{5}=B^{4} \times S^{1}$ where $B^{4}$ is a four-dimensional ball with $\partial B^{4}=S^{3}$. After some calculation, we obtain the following CS action,

$$
W_{\mathrm{CS}}=\left(\frac{2(5 a-3 c)}{27 \pi^{2}} \Delta^{3}+\frac{2(a-c)}{3} \Delta\right) \frac{1}{\beta^{2}} \int_{S^{3}} \mathbf{a} d \mathbf{a}-\frac{a-c}{3(2 \pi)^{2}} \Delta \int_{S^{3}} \operatorname{tr}\left[\hat{\boldsymbol{\Gamma}} d \hat{\boldsymbol{\Gamma}}+\frac{2}{3} \hat{\boldsymbol{\Gamma}}^{3}\right]
$$

There are only gauge and mixed CS terms on $S^{3}$. After integrating over $S^{3}$, we obtain the following results,

$$
\begin{aligned}
\frac{1}{\beta^{2}} \int_{S^{3}} \mathbf{a} d \mathbf{a} & =-\frac{(2 \pi)^{2} \omega_{1} \omega_{2}}{\left(\frac{\beta^{2}}{r^{2}}-\omega_{1}^{2}\right)\left(\frac{\beta^{2}}{r^{2}}-\omega_{2}^{2}\right)}=-\frac{4 \pi^{2}}{\omega_{1} \omega_{2}} \cdot\left[1+\mathcal{O}\left(\frac{\beta^{2}}{r^{2} \omega^{2}}\right)\right] \\
\int_{S^{3}} \operatorname{tr}\left[\hat{\boldsymbol{\Gamma}} d \hat{\boldsymbol{\Gamma}}+\frac{2}{3} \hat{\boldsymbol{\Gamma}}^{3}\right] & =\frac{\beta^{2}}{r^{2}} \frac{16 \pi^{2} \omega_{1} \omega_{2}}{\left(\frac{\beta^{2}}{r^{2}}-\omega_{1}^{2}\right)\left(\frac{\beta^{2}}{r^{2}}-\omega_{2}^{2}\right)}=\mathcal{O}\left(\frac{\beta^{2}}{r^{2} \omega^{2}}\right) .
\end{aligned}
$$

Therefore in the scaling limit (2.27), the Cardy free energy becomes

$$
\log I=-\lim _{\beta \rightarrow 0} W_{\mathrm{CS}}+\mathcal{O}(\log \omega)=\frac{8 \Delta^{3}}{27 \omega_{1} \omega_{2}}(5 a-3 c)+\frac{8 \pi^{2} \Delta}{3 \omega_{1} \omega_{2}}(a-c)+\mathcal{O}(\log \omega)
$$

It is exactly the same with the Cardy formula given in [37, 38].

The Cardy formula (A.12) can also be obtained from the equivariant integral of the thermal anomaly polynomial (A.8) as follows,

$$
\log I=-\int P_{6}^{T}+\mathcal{O}(\log \omega)
$$

Now, we use the Duistermaat-Heckman formula (4.2) to evaluate the equivariant integral. The 0 -form components of the equivariant classes at the fixed point $p_{0}$ are given as follows,

$$
c_{1}(R)_{p_{0}}=-\Delta,\left.\quad p_{1}(T)\right|_{p_{0}}=\omega_{1}^{2}+\omega_{2}^{2},\left.\quad e(T M)\right|_{p_{0}}=\omega_{1} \omega_{2},\left.\quad \frac{\mathbf{F}_{T}^{2}}{4 \pi^{2}}\right|_{p_{0}}=-(2 \pi i)^{2}
$$




\begin{tabular}{|c|c|c|c|c|c|c|c|c|}
\hline multiplet & letter & type & $E$ & $Q_{R}$ & $J_{1}$ & $J_{2}$ & $J_{3}$ & letter index \\
\hline half-hyper & $\phi$ & $\mathrm{B}$ & 2 & $1 / 2$ & 0 & 0 & 0 & $(-1) q_{1} q_{2} q_{3}$ \\
\hline tensor & $B_{\mu \nu}^{+}$ & $\mathrm{B}$ & 3 & 0 & 1 & 1 & 1 & $\left(q_{1} q_{2} q_{3}\right)^{2}$ \\
\hline tensor & $\xi^{+}$ & $\mathrm{F}$ & $5 / 2$ & $1 / 2$ & $1 / 2$ & $1 / 2$ & $-1 / 2$ & $(-1)\left(q_{1} q_{2}\right)^{2}$ \\
\hline tensor & $\xi^{+}$ & $\mathrm{F}$ & $5 / 2$ & $1 / 2$ & $1 / 2$ & $-1 / 2$ & $1 / 2$ & $(-1)\left(q_{1} q_{3}\right)^{2}$ \\
\hline tensor & $\xi^{+}$ & $\mathrm{F}$ & $5 / 2$ & $1 / 2$ & $-1 / 2$ & $1 / 2$ & $1 / 2$ & $(-1)\left(q_{2} q_{3}\right)^{2}$ \\
\hline derivative & $\partial_{1}$ & $\mathrm{~B}$ & 1 & 0 & 1 & 0 & 0 & $q_{1}^{2}$ \\
\hline derivative & $\partial_{2}$ & $\mathrm{~B}$ & 1 & 0 & 0 & 1 & 0 & $q_{2}^{2}$ \\
\hline derivative & $\partial_{3}$ & $\mathrm{~B}$ & 1 & 0 & 0 & 0 & 1 & $q_{3}^{2}$ \\
\hline
\end{tabular}

Table 3. BPS letters in $6 \mathrm{~d}$ multiplets. Type denotes a boson/fermion statistics, and $f$ denotes a single particle index of the BPS letter. The fugacities are defined as $q_{I}=e^{-\omega_{I} / 2}$. Note that $(-1)$ in the single particle index should be also treated as a fugacity.

Then, we obtain

$$
-\int P_{6}^{T}=\frac{8 \Delta^{3}}{27 \omega_{1} \omega_{2}}(5 a-3 c)+\frac{8 \pi^{2} \Delta}{3 \omega_{1} \omega_{2}}(a-c)-\frac{2 \Delta\left(\omega_{1}^{2}+\omega_{2}^{2}\right)}{3 \omega_{1} \omega_{2}}(a-c) .
$$

As a result, the Cardy formula (A.12) can be obtained from (A.15) by our relation (A.13). In this case, $\mathcal{O}\left(\omega^{0}\right)$ term in (A.15) can be ignored since it is subleading than $\mathcal{O}(\log \omega)$.

\section{B Modified index of $6 \mathrm{~d}$ supermultiplets}

In this section, we compute the modified superconformal indices of free $(1,0)$ tensor and hypermultiplets defined as follows,

$$
I=\operatorname{Tr}\left[e^{-2 \pi i Q_{R}} \cdot e^{-\omega_{1}\left(J_{1}+Q_{R}\right)-\omega_{2}\left(J_{2}+Q_{R}\right)-\omega_{3}\left(J_{3}+Q_{R}\right)}\right] .
$$

Note that $\Delta_{R}$ in (3.11) is absorbed by the chemical potential relation $\Delta_{R}-\omega_{1}-\omega_{2}-\omega_{3}=$ $2 \pi i$. More detailed explanation is given in section 3 .

6d $(1,0)$ hypermultiplet has component fields $\left(\phi, \psi^{+}\right)$where $\phi$ is a complex scalar in $(\mathbf{2}, \mathbf{1})$ representation of $\mathrm{SU}(2)_{R} \times \mathrm{SO}(6)$, and $\psi^{+}$is a fermion in $(\mathbf{1}, \mathbf{4})$. Tensor multiplet has component fields $\left(a, \xi^{+}, B_{\mu \nu}^{+}\right)$where $a$ is a real scalar in $(\mathbf{1}, \mathbf{1}), \xi^{+}$is a fermion in $(\mathbf{2}, \mathbf{4})$, and $B_{\mu \nu}^{+}$is a self-dual tensor in $(\mathbf{1}, \mathbf{1 0})$. The charges of the BPS fields that saturate the BPS bound $E \geq J_{1}+J_{2}+J_{3}+4 Q_{R}$ are listed in the table 3 .

The superconformal index can be obtained from the plethystic exponential of the BPS letters as follows,

$$
I=\exp \sum_{n=1}^{\infty} \frac{1}{n}\left[f_{B}\left(\bullet^{n}\right)-(-1)^{n} f_{F}\left(\bullet^{n}\right)\right]
$$

where $f_{B}$ is a single particle index of bosonic fields and $f_{B}$ is for fermionic fields. Here, collectively denotes the fugacity variables. Then, the modified superconformal indices of 
$(1,0)$ supermultiplets are given as follows,

$$
\begin{aligned}
I_{\text {hyp }} & =\exp \sum_{n=1}^{\infty} \frac{2}{n} \frac{\left(-q_{1} q_{2} q_{3}\right)^{n}}{\left(1-q_{1}^{2 n}\right)\left(1-q_{2}^{2 n}\right)\left(1-q_{3}^{2 n}\right)} \\
I_{\text {ten }} & =\exp \sum_{n=1}^{\infty} \frac{1}{n} \frac{\left(q_{1} q_{2} q_{3}\right)^{2 n}-\left(q_{1} q_{2}\right)^{2 n}-\left(q_{2} q_{3}\right)^{2 n}-\left(q_{3} q_{1}\right)^{2 n}}{\left(1-q_{1}^{2 n}\right)\left(1-q_{2}^{2 n}\right)\left(1-q_{3}^{2 n}\right)}
\end{aligned}
$$

where fugacities are defined as $q_{I}=e^{-\omega_{I} / 2}$. Note that by shifting $\omega_{3} \rightarrow \omega_{3}-2 \pi i$ which is equivalent to shifting $q_{3} \rightarrow-q_{3}$, we obtain the original superconformal indices of tensor/hypermultiplets computed in [57].

Open Access. This article is distributed under the terms of the Creative Commons Attribution License (CC-BY 4.0), which permits any use, distribution and reproduction in any medium, provided the original author(s) and source are credited.

\section{References}

[1] J. Kinney, J.M. Maldacena, S. Minwalla and S. Raju, An index for 4 dimensional super conformal theories, Commun. Math. Phys. 275 (2007) 209 [hep-th/0510251] [INSPIRE].

[2] C. Romelsberger, Counting chiral primaries in $N=1, d=4$ superconformal field theories, Nucl. Phys. B 747 (2006) 329 [hep-th/0510060] [INSPIRE].

[3] J. Bhattacharya, S. Bhattacharyya, S. Minwalla and S. Raju, Indices for superconformal field theories in 3,5 and 6 dimensions, JHEP 02 (2008) 064 [arXiv:0801.1435] [INSPIRE].

[4] H.-C. Kim and S. Kim, M5-branes from gauge theories on the 5-sphere, JHEP 05 (2013) 144 [arXiv: 1206.6339] [INSPIRE].

[5] J. Kallen, J.A. Minahan, A. Nedelin and M. Zabzine, $N^{3}$-behavior from $5 D$ Yang-Mills theory, JHEP 10 (2012) 184 [arXiv:1207.3763] [INSPIRE].

[6] H.-C. Kim and K. Lee, Supersymmetric M5 brane theories on $R \times C P^{2}$, JHEP 07 (2013) 072 [arXiv: 1210.0853] [INSPIRE].

[7] G. Lockhart and C. Vafa, Superconformal partition functions and non-perturbative topological strings, JHEP 10 (2018) 051 [arXiv: 1210.5909] [INSPIRE].

[8] H.-C. Kim, J. Kim and S. Kim, Instantons on the 5-sphere and M5-branes, arXiv: 1211.0144 [INSPIRE].

[9] J.A. Minahan, A. Nedelin and M. Zabzine, 5D super Yang-Mills theory and the correspondence to $A d S_{7} / C F T_{6}$, J. Phys. A 46 (2013) 355401 [arXiv:1304.1016] [InSPIRE].

[10] H.-C. Kim, S. Kim, S.-S. Kim and K. Lee, The general M5-brane superconformal index, arXiv: 1307.7660 [INSPIRE].

[11] S. Kim and K. Lee, Indices for 6 dimensional superconformal field theories, J. Phys. A 50 (2017) 443017 [arXiv: 1608. 02969] [INSPIRE].

[12] I.R. Klebanov and A.A. Tseytlin, Entropy of near extremal black p-branes, Nucl. Phys. B 475 (1996) 164 [hep-th/9604089] [INSPIRE].

[13] D. Freed, J.A. Harvey, R. Minasian and G.W. Moore, Gravitational anomaly cancellation for M-theory five-branes, Adv. Theor. Math. Phys. 2 (1998) 601 [hep-th/9803205] [INSPIRE]. 
[14] K. Ohmori, H. Shimizu, Y. Tachikawa and K. Yonekura, Anomaly polynomial of general $6 d$ SCFTs, PTEP 2014 (2014) 103B07 [arXiv: 1408.5572] [INSPIRE].

[15] K. Intriligator, $6 d, N=(1,0)$ Coulomb branch anomaly matching, JHEP 10 (2014) 162 [arXiv: 1408.6745] [INSPIRE].

[16] N. Bobev, M. Bullimore and H.-C. Kim, Supersymmetric Casimir energy and the anomaly polynomial, JHEP 09 (2015) 142 [arXiv:1507.08553] [INSPIRE].

[17] H.-C. Kim, S. Kim, E. Koh, K. Lee and S. Lee, On instantons as Kaluza-Klein modes of M5-branes, JHEP 12 (2011) 031 [arXiv:1110.2175] [INSPIRE].

[18] S. Kim and J. Nahmgoong, Asymptotic M5-brane entropy from S-duality, JHEP 12 (2017) 120 [arXiv: 1702.04058] [INSPIRE].

[19] S.M. Hosseini, K. Hristov and A. Zaffaroni, An extremization principle for the entropy of

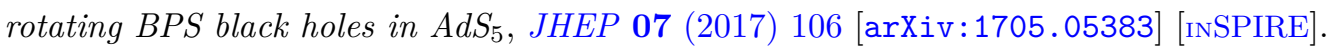

[20] S.M. Hosseini, K. Hristov and A. Zaffaroni, A note on the entropy of rotating BPS $A d S_{7} \times S^{4}$ black holes, JHEP 05 (2018) 121 [arXiv:1803.07568] [INSPIRE].

[21] S. Choi, C. Hwang, S. Kim and J. Nahmgoong, Entropy functions of BPS black holes in $A d S_{4}$ and $A d S_{6}$, J. Korean Phys. Soc. 76 (2020) 101 [arXiv:1811.02158] [INSPIRE].

[22] S. Choi, C. Hwang and S. Kim, Quantum vortices, M2-branes and black holes, arXiv: 1908.02470 [INSPIRE].

[23] J. Nian and L.A. Pando Zayas, Microscopic entropy of rotating electrically charged $A d S_{4}$ black holes from field theory localization, JHEP 03 (2020) 081 [arXiv: 1909. 07943] [INSPIRE].

[24] S. Choi and C. Hwang, Universal 3d Cardy block and black hole entropy, JHEP 03 (2020) 068 [arXiv: 1911.01448] [INSPIRE].

[25] A. Cabo-Bizet, D. Cassani, D. Martelli and S. Murthy, Microscopic origin of the Bekenstein-Hawking entropy of supersymmetric AdS $S_{5}$ black holes, JHEP 10 (2019) 062 [arXiv: 1810.11442] [INSPIRE].

[26] S. Choi, J. Kim, S. Kim and J. Nahmgoong, Large AdS black holes from QFT, arXiv:1810.12067 [INSPIRE].

[27] F. Benini and P. Milan, Black holes in $4 D N=4$ super-Yang-Mills field theory, Phys. Rev. $X$ 10 (2020) 021037 [arXiv: 1812.09613] [INSPIRE].

[28] S. Choi and S. Kim, Large AdS $S_{6}$ black holes from $C F T_{5}$, arXiv:1904.01164 [INSPIRE].

[29] S. Choi, J. Kim, S. Kim and J. Nahmgoong, Comments on deconfinement in AdS/CFT, arXiv:1811.08646 [INSPIRE].

[30] D. Cassani and L. Papini, The BPS limit of rotating AdS black hole thermodynamics, JHEP 09 (2019) 079 [arXiv: 1906. 10148] [inSPIRE].

[31] G. Kántor, C. Papageorgakis and P. Richmond, AdS $S_{7}$ black-hole entropy and $5 D N=2$ Yang-Mills, JHEP 01 (2020) 017 [arXiv:1907.02923] [INSPIRE].

[32] J.L. Cardy, Operator content of two-dimensional conformally invariant theories, Nucl. Phys. B 270 (1986) 186 [INSPIRE].

[33] A. Strominger and C. Vafa, Microscopic origin of the Bekenstein-Hawking entropy, Phys. Lett. B 379 (1996) 99 [hep-th/9601029] [INSPIRE]. 
[34] L. Di Pietro and Z. Komargodski, Cardy formulae for SUSY theories in $d=4$ and $d=6$, JHEP 12 (2014) 031 [arXiv: 1407.6061] [INSPIRE].

[35] M. Honda, Quantum black hole entropy from $4 d$ supersymmetric Cardy formula, Phys. Rev. D 100 (2019) 026008 [arXiv:1901.08091] [INSPIRE].

[36] A. Arabi Ardehali, Cardy-like asymptotics of the $4 d N=4$ index and AdS $S_{5}$ blackholes, JHEP 06 (2019) 134 [arXiv:1902.06619] [INSPIRE].

[37] J. Kim, S. Kim and J. Song, A 4d $N=1$ Cardy formula, JHEP 01 (2021) 025 [arXiv: 1904.03455] [INSPIRE].

[38] A. Cabo-Bizet, D. Cassani, D. Martelli and S. Murthy, The asymptotic growth of states of the $4 d N=1$ superconformal index, JHEP 08 (2019) 120 [arXiv: 1904.05865] [INSPIRE].

[39] C.-M. Chang, M. Fluder, Y.-H. Lin and Y. Wang, Proving the $6 d$ Cardy formula and matching global gravitational anomalies, arXiv:1910.10151 [INSPIRE].

[40] K. Jensen, R. Loganayagam and A. Yarom, Thermodynamics, gravitational anomalies and cones, JHEP 02 (2013) 088 [arXiv:1207.5824] [INSPIRE].

[41] K. Jensen, R. Loganayagam and A. Yarom, Anomaly inflow and thermal equilibrium, JHEP 05 (2014) 134 [arXiv: 1310.7024] [INSPIRE].

[42] K. Jensen, R. Loganayagam and A. Yarom, Chern-Simons terms from thermal circles and anomalies, JHEP 05 (2014) 110 [arXiv:1311.2935] [INSPIRE].

[43] S. Yankielowicz and Y. Zhou, Supersymmetric Rényi entropy and anomalies in $6 d(1,0)$ SCFTs, JHEP 04 (2017) 128 [arXiv:1702.03518] [INSPIRE].

[44] G. 't Hooft, On the phase transition towards permanent quark confinement, Nucl. Phys. B 138 (1978) 1 [INSPIRE].

[45] J. Wess and B. Zumino, Consequences of anomalous Ward identities, Phys. Lett. B 37 (1971) 95 [INSPIRE].

[46] A. Bilal, Lectures on anomalies, arXiv:0802.0634 [INSPIRE].

[47] W.A. Bardeen and B. Zumino, Consistent and covariant anomalies in gauge and gravitational theories, Nucl. Phys. B 244 (1984) 421 [InSPIRE].

[48] W. Nahm, Supersymmetries and their representations, Nucl. Phys. B 135 (1978) 149 [INSPIRE].

[49] E. Witten, Some comments on string dynamics, in Strings 95: future perspectives in string theory, (1995), pg. 501 [hep-th/9507121] [INSPIRE].

[50] N. Seiberg and E. Witten, Comments on string dynamics in six-dimensions, Nucl. Phys. B 471 (1996) 121 [hep-th/9603003] [INSPIRE].

[51] A. Strominger, Open p-branes, Phys. Lett. B 383 (1996) 44 [hep-th/9512059] [inSPIRE].

[52] S. Fredenhagen and V. Schomerus, Boundary Liouville theory at $c=1$, JHEP 05 (2005) 025 [hep-th/0409256] [INSPIRE].

[53] J.J. Heckman, D.R. Morrison, T. Rudelius and C. Vafa, Atomic classification of $6 D$ SCFTs, Fortsch. Phys. 63 (2015) 468 [arXiv:1502.05405] [InSPIRE].

[54] P. Hořava and E. Witten, Heterotic and type-I string dynamics from eleven-dimensions, Nucl. Phys. B 460 (1996) 506 [hep-th/9510209] [INSPIRE]. 
[55] A. Klemm, P. Mayr and C. Vafa, BPS states of exceptional noncritical strings, Nucl. Phys. B Proc. Suppl. 58 (1997) 177 [hep-th/9607139] [INSPIRE].

[56] M. Del Zotto, J.J. Heckman, A. Tomasiello and C. Vafa, 6d conformal matter, JHEP 02 (2015) 054 [arXiv: 1407.6359] [inSPIRE].

[57] S. Benvenuti, G. Bonelli, M. Ronzani and A. Tanzini, Symmetry enhancements via $5 d$ instantons, $q \mathcal{W}$-algebrae and $(1,0)$ superconformal index, JHEP 09 (2016) 053 [arXiv: 1606.03036] [INSPIRE].

[58] J.J. Duistermaat and G.J. Heckman, On the variation in the cohomology of the symplectic form of the reduced phase space, Invent. Math. 69 (1982) 259.

[59] F. Apruzzi, M. Fazzi, D. Rosa and A. Tomasiello, All AdS $S_{7}$ solutions of type-II supergravity, JHEP 04 (2014) 064 [arXiv: 1309.2949] [INSPIRE].

[60] J.M. Maldacena, The large $N$ limit of superconformal field theories and supergravity, Int. J. Theor. Phys. 38 (1999) 1113 [hep-th/9711200] [INSPIRE].

[61] M. Cvetič, G.W. Gibbons, H. Lü and C.N. Pope, Rotating black holes in gauged supergravities: thermodynamics, supersymmetric limits, topological solitons and time machines, hep-th/0504080 [INSPIRE].

[62] K. Lee and J. Nahmgoong, Cardy limits of 6d superconformal theories, arXiv:2006.10294 [INSPIRE].

[63] Z. Duan and J. Nahmgoong, Bootstrapping ADE M-strings, arXiv:2009.03626 [INSPIRE].

[64] A. Arabi Ardehali, High-temperature asymptotics of the 4d superconformal index, Ph.D. thesis, Michigan U., Ann Arbor, MI, U.S.A. (2016) [arXiv: 1605.06100] [InSPIRE].

[65] M. Beccaria and A.A. Tseytlin, Conformal anomaly c-coefficients of superconformal $6 d$ theories, JHEP 01 (2016) 001 [arXiv: 1510.02685] [INSPIRE].

[66] D. Anselmi, D.Z. Freedman, M.T. Grisaru and A.A. Johansen, Nonperturbative formulas for central functions of supersymmetric gauge theories, Nucl. Phys. B 526 (1998) 543 [hep-th/9708042] [INSPIRE]. 